\title{
Persistent digestive disorders in the tropics: causative infectious pathogens and reference diagnostic tests
}

\author{
Sören L Becker ${ }^{1,2,3}$, Jürg Vogt ${ }^{1,2}$, Stefanie Knopp ${ }^{1,2}$, Marcus Panning ${ }^{4}$, David C Warhurst ${ }^{5}$, Katja Polman ${ }^{6}$, \\ Hanspeter Marti ${ }^{2,7}$, Lutz von Müller ${ }^{3}$, Cedric P Yansouni ${ }^{8,9}$, Jan Jacobs ${ }^{9}$, Emmanuel Bottieau ${ }^{9}$, Moussa Sacko ${ }^{10}$, \\ Suman Rijal ${ }^{11}$, Fransiska Meyanti ${ }^{12}$, Michael A Miles ${ }^{5}$, Marleen Boelaert ${ }^{13}$, Pascal Lutumba ${ }^{14,15}$, \\ Lisette van Lieshout ${ }^{16}$, Eliézer K N'Goran ${ }^{17,18}$, François Chappuis ${ }^{19}$ and Jürg Utzinger ${ }^{1,2^{*}}$
}

\begin{abstract}
Background: Persistent digestive disorders account for considerable disease burden in the tropics. Despite advances in understanding acute gastrointestinal infections, important issues concerning epidemiology, diagnosis, treatment and control of most persistent digestive symptomatologies remain to be elucidated. Helminths and intestinal protozoa are considered to play major roles, but the full extent of the aetiologic spectrum is still unclear. We provide an overview of pathogens causing digestive disorders in the tropics and evaluate available reference tests.

Methods: We searched the literature to identify pathogens that might give rise to persistent diarrhoea, chronic abdominal pain and/or blood in the stool. We reviewed existing laboratory diagnostic methods for each pathogen and stratified them by (i) microscopy; (ii) culture techniques; (iii) immunological tests; and (iv) molecular methods. Pathogen-specific reference tests providing highest diagnostic accuracy are described in greater detail.

Results: Over 30 pathogens may cause persistent digestive disorders. Bacteria, viruses and parasites are important aetiologic agents of acute and long-lasting symptomatologies. An integrated approach, consisting of stool culture, microscopy and/or specific immunological techniques for toxin, antigen and antibody detection, is required for accurate diagnosis of bacteria and parasites. Molecular techniques are essential for sensitive diagnosis of many viruses, bacteria and intestinal protozoa, and are increasingly utilised as adjuncts for helminth identification.

Conclusions: Diagnosis of the broad spectrum of intestinal pathogens is often cumbersome. There is a need for rapid diagnostic tests that are simple and affordable for resource-constrained settings, so that the management of patients suffering from persistent digestive disorders can be improved.
\end{abstract}

Keywords: Bacteria, Clinical microbiology, Diagnosis, Digestive disorders, Gastroenterology, Helminths, Intestinal protozoa, Persistent diarrhoea, Virus

\section{Background}

Diarrhoeal diseases and other digestive disorders are leading causes of morbidity and mortality worldwide, with the highest burden concentrated in tropical and subtropical areas that often lack access to clean water and adequate sanitation, and where hygienic conditions

\footnotetext{
* Correspondence: juerg.utzinger@unibas.ch

'Department of Epidemiology and Public Health, Swiss Tropical and Public Health Institute, Basel, Switzerland

${ }^{2}$ University of Basel, Basel, Switzerland

Full list of author information is available at the end of the article
}

are generally poor [1]. According to the World Health Organization (WHO), diarrhoea is classified into three different categories, namely (i) acute watery diarrhoea (lasting several hours or days); (ii) acute bloody diarrhoea (synonymous: dysentery); and (iii) persistent diarrhoea (lasting 14 days or longer) [2]. 'Chronic diarrhoea' is often referred to as an individual term applicable to diarrhoea lasting more than 4-6 weeks, but it still lacks an unambiguous definition.

With an estimated burden of 89.5 million disabilityadjusted life years (DALYs) caused in 2010, diarrhoeal

\section{Biomed Central}


diseases rank fourth in the recently published Global Burden of Disease Study [3]. Acute diarrhoeal episodes are mainly due to bacterial and viral pathogens that may cause a variety of clinical syndromes ranging from selflimiting events to life-threatening diseases. Children are most vulnerable and diarrhoeal diseases were responsible for more than 1.4 million deaths in 2010, ranking this disorder at position seven on the main causes of death [4]. In the last decades, concerted efforts have considerably improved our understanding of the epidemiology, diagnosis, treatment and control of many diarrhoeagenic pathogens globally, for instance due to the introduction of rotavirus vaccination programmes in many countries since 2006 [5]. As a result, mortality due to diarrhoeal diseases has been reduced from an estimated 2.5 million in 1990 to just under 1.5 million in 2010, a decrease of $42 \%$ [4]. However, few research activities have focused on the investigation of persistent diarrhoea and non-acute abdominal pain due to digestive disorders in the tropics. Hence, little is known regarding its aetiology, epidemiology and disease burden. It is widely acknowledged that intestinal parasites, particularly helminths and intestinal protozoa play a major role as causative agents of persistent digestive symptomatologies [6].

Infections with helminths and intestinal protozoa belong to the neglected tropical diseases, along with other diseases caused by bacterial (e.g. Buruli ulcer), viral (e.g. dengue) and fungal infections (e.g. mycetoma) [7]. More than 5 billion people are at risk of neglected tropical diseases, with the common soil-transmitted helminths (i.e. Ascaris lumbricoides, hookworm and Trichuris trichiura), exhibiting the widest geographical distribution [8]. Due to their intimate connection with poverty, the highest prevalences of neglected tropical diseases are observed in remote rural and deprived urban settings in the developing world $[7,9,10]$. Neglected tropical diseases drain the social and economic development in endemic countries and they negatively impact on people's quality of life and well-being at all levels [11-15].

A major challenge in the clinical management of persistent digestive disorders is the weakness of health systems in many low-income countries [16-18]. Hence, affected people might only seek care at a late stage in their therapeutic itinerary, usually at primary health care centres $[19,20]$. However, these primary health care centres are often under-staffed and ill-equipped, resulting in a low quality of care. The causes of persistent diarrhoea and other digestive disorders are frequently misdiagnosed due to the often unspecific clinical presentations and the absence of evidence-based algorithms for in-depth investigation [7,21]. The notorious underfinancing of health systems in many tropical and subtropical countries explains the severe neglect of laboratory networks and the only limited availability of basic tests for diagnostic services (e.g. direct faecal smears for helminth diagnosis or blood films for malaria diagnosis) [22]. Hence, in many developing countries, current diagnostic and treatment algorithms are often empirical, whereas local prevalence data and differential diagnoses are rarely taken into account at the primary care level.

Against this background, NIDIAG, an international collaboration on integrated diagnosis-treatment platforms, funded by the European Commission, sets out to develop an improved system for delivering primary health care in resource-constrained settings and proposes an integrated approach to this challenge. Emphasis is placed on a patient-centred approach starting from the presentation at the primary health care level of a clinical syndrome that might be due to 'common' pathogens. Three clinical syndromes will be investigated in the NIDIAG framework, namely (1) neurological disorders [23]; (2) persistent fever [24]; and (3) digestive disorders. Here, we focus on digestive disorders, which we define as (i) persistent ( $\geq 2$ weeks) abdominal pain; (ii) persistent $(\geq 2$ weeks) diarrhoea (dysenteric or non-dysenteric); and/or (iii) blood in the stool. These digestive disorders will be investigated at different study sites in tropical areas of Africa (Côte d'Ivoire and Mali) and Asia (Indonesia and Nepal). Before clinically applicable diagnosis-treatment algorithms can be developed, the following major challenges/open issues have to be addressed. Firstly, few studies analysed the spectrum of intestinal pathogens causing persistent digestive disorders in the tropics. Therefore, epidemiological investigations targeting all potential pathogens are desirable to define the most common bacteria, parasites and viruses in the different study settings. Secondly, most diagnostic tests have only been validated in Western settings, and hence their diagnostic accuracy in the tropics remains to be determined.

In this manuscript, pursuing an extensive literature review complemented with expert opinion, we provide an overview of potential pathogens (bacterial, parasitic and viral) that might give rise to digestive disorders as defined above. Available diagnostic tests for the identified pathogens are summarised and reviewed, and we propose pathogen-specific reference tests to be utilised for an in-depth diagnostic work-up of symptomatic patients in the different study sites.

\section{Methods}

\section{Framework}

A symptomatology according to the aforementioned inclusion criteria for the syndrome of digestive disorders is likely to be caused by a large variety of infections, but also non-infectious diseases. For example, blood in the stool, accompanied by persistent abdominal pain, may be indicative of colorectal carcinoma or inflammatory disorders (e.g. Crohn's disease and ulcerative colitis), but 
may also be a sign of Schistosoma mansoni (a helminth) or Entamoeba histolytica (an intestinal protozoon) infection. The aim of the NIDIAG project is to develop evidence-based diagnosis-treatment algorithms that can easily be applied in resource-constrained health care settings. As neither diagnosis nor treatment of many noninfectious diseases are currently feasible in remote rural areas of most developing countries, only infectious aetiologies of digestive disorders that may cause severe disease and that are treatable will be thoroughly assessed within the frame of the NIDIAG project and were therefore prioritised in our literature search.

\section{Search strategy, data extraction and analysis}

We performed a literature review to identify and define the bacterial, parasitic and viral pathogens that may give rise to persistent diarrhoea and chronic digestive disorders, and to obtain information on their respective diagnostic methods in order to describe appropriate reference laboratory tests. Since the role of fungi as causative pathogens of gastrointestinal infections is still under debate, fungal infections were not included in this review [25]. The available literature was reviewed by three independent groups. The results were compared, discussed and finally synthesised. Additionally, a number of experts were consulted to complement the literature review.

In a first step, we examined a series of textbooks pertaining to medical bacteriology, parasitology and virology. Moreover, we searched the electronic database MEDLINE/PubMed for infectious pathogens that may cause digestive disorders as defined in the inclusion criteria. After having identified a set of more than 30 pathogens, we searched the database with the following search term for all infectious agents: "disease name/[Mesh]" and the subheading "diagnosis" (e.g. "ascariasis/diagnosis" [Mesh]). The focus of the MEDLINE/PubMed search was on established laboratory tests as well as on newer diagnostic methods, which have been validated recently or are currently under validation (e.g. studies objectively assessing the diagnostic accuracy of different tests). Hence, we primarily focused on reviews, comparative studies and evaluation studies. Our search had no language or other restrictions and we included studies that were published until mid-April 2012.

\section{Results}

Our literature review revealed more than 30 bacterial, parasitic and viral pathogens that may cause persistent digestive disorders. Many of these infectious agents are epidemiologically well characterised in Western settings, while data regarding their occurrence in tropical and subtropical areas are scarce and often contradictory [6,26-28]. Table 1 provides a list of all selected pathogens and typical clinical characteristics that may assist clinicians to curtail their differential diagnosis. However, pathogen-specific diagnosis can rarely be done based on the clinical presentations, and hence additional diagnostic tools are needed.

The large number of available diagnostic tests for the selected pathogens is a challenge for providing the single most accurate method for a given pathogen. Hence, we classified the different methods into four diagnostic categories, namely (i) microscopy; (ii) culture; (iii) immunology (including enzyme immunoassays (EIA), serotyping of isolates and serology); and (iv) molecular biological diagnosis (e.g. polymerase chain reaction (PCR) assays and DNA sequencing). Selection of a reference test for each specific pathogen is primarily based on the sensitivity and specificity of the test as well as practical considerations (e. g. costs, ease of application, availability, etc.). The results are presented in Table 2 (bacteria), Table 3 (intestinal protozoa), Table 4 (helminths) and Table 5 (viruses). Specific issues on the diagnostic work-up are summarised in the following sub-chapters.

\section{Bacterial pathogens}

Aeromonas spp., Campylobacter jejuni, C. coli, Plesiomonas shigelloides, Salmonella enterica (non-typhoidal serovars, e. g. S. enterica ser. Enteritidis, S. enterica ser. Typhimurium), Shigella spp., Vibrio spp., Yersinia enterocolitica, $Y$. pseudotuberculosis

A stool culture on selective media is the test of choice to detect these diarrhoeagenic bacteria [31,48,50,52,53]. Different solid media (e.g. selective agar plates containing antibiotics and substances favouring the growth of the sought microorganism) are inoculated with a stool specimen to detect and isolate enteric pathogens. The additional use of a selective enrichment broth is helpful to identify pathogenic bacteria if their presence is quantitatively so low that they might otherwise be overlooked on solid media due to the overgrowth of non-pathogenic intestinal flora. The inoculated media are usually incubated for $24-72$ hours at $35^{\circ} \mathrm{C}$ at ambient atmosphere to allow the bacteria to form macroscopically visible colonies. Of note, Campylobacter spp. are isolated using different growth conditions, i.e. incubation at a higher temperature of $42^{\circ} \mathrm{C}$ in microaerophilic atmosphere [53].

Following the incubation period, the agar plates are examined and morphologically suspicious colonies are identified using different biochemical identification panels or automated phenotypic identification systems (e.g. Vitek ${ }^{\circledR}$; bioMérieux, Marcy l'Étoile, France). Recently, more rapid identification algorithms making use of mass spectrometry (MS) have successfully been implemented into clinical microbiology laboratories (e.g. matrix-assisted laser desorption ionization time-of-flight (MALDI-TOF) MS (e.g. MicroFlex LT; Bruker Daltonics, Bremen, Germany) [98].

Stool culture remains the diagnostic 'gold' standard for enteric pathogenic bacteria disposing certain characteristics 
Table 1 Overview of intestinal pathogens (bacteria, intestinal protozoa, helminths and viruses) that may cause persistent digestive disorders in infected individuals

\begin{tabular}{llll}
\hline Enteric pathogen & $\begin{array}{l}\text { Persistent } \\
\text { diarrhoea }\end{array}$ & $\begin{array}{l}\text { Persistent } \\
\text { abdominal } \\
\text { pain }\end{array}$ & $\begin{array}{l}\text { Blood in } \\
\text { the stool }\end{array}$ \\
& &
\end{tabular}

\section{Bacteria}

Aeromonas spp.

Campylobacter jejuni, C. coli

Clostridium difficile

Escherichia coli

Enteroaggregative E. coli + (EAEC)

Enteropathogenic E. coli (EPEC)

Enteroinvasive E. coli (EIEC)

Enterohaemorrhagic E. coli $\quad+$ (STEC/EHEC)

Enterotoxigenic E. coli (ETEC) +

Diffusely adherent E. coli + (DAEC)

Mycobacterium tuberculosis and atypical mycobacteria

Plesiomonas shigelloides

Salmonella enterica (typhoidal and non-typhoidal serovars)

Shigella spp.

Tropheryma whipplei

Vibrio spp.

Yersinia enterocolitica,

Y. pseudotuberculosis

Intestinal protozoa

Balantidium coli

Blastocystis hominis ${ }^{a}$

Cryptosporidium spp.

Cyclospora cayetanensis

Dientamoeba fragilis ${ }^{\mathrm{a}}$

Entamoeba histolytica

Giardia intestinalis (syn:: G. lamblia and G. duodenalis)

Isospora belli (syn.: Cystoisospora + belli)

Species of microsporidia

Helminths

Cestodes

Diphyllobothrium latum
Hymenolepis spp.
Taenia spp.

Nematodes

Ascaris lumbricoides
$+$

$+$

$+$

$+$

.

$+$

$+$

$+$

$+$

$+$

$+$

$+$

$+$

$+$

(+)

$+$

$+$

$+$

$+$

$+$

(+)

$+$

$-$

$+$

$+$

$+$

(+)

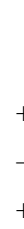

-
+
+

Table 1 Overview of intestinal pathogens (bacteria, intestinal protozoa, helminths and viruses) that may cause persistent digestive disorders in infected individuals (Continued)

\begin{tabular}{|c|c|c|c|}
\hline Capillaria philippinensis & + & + & - \\
\hline $\begin{array}{l}\text { Hookworm (Ancylostoma } \\
\text { duodenale and Necator } \\
\text { americanus) }\end{array}$ & + & + & - \\
\hline Strongyloides stercoralis & + & + & $(+)$ \\
\hline Trichuris trichiura & + & + & - \\
\hline \multicolumn{4}{|l|}{ Trematodes } \\
\hline Intestinal flukes & + & + & - \\
\hline $\begin{array}{l}\text { Intestinal blood flukes: } \\
\text { Schistosoma mansoni, } \\
\text { S. intercalatum, S. japonicum, } \\
\text { S. mekongi }\end{array}$ & + & + & + \\
\hline \multicolumn{4}{|l|}{ Viruses } \\
\hline Adenovirus & + & - & - \\
\hline Astrovirus & $(+)$ & - & - \\
\hline Bocavirus & $(+)$ & - & - \\
\hline Coronavirus & $(+)$ & - & - \\
\hline Cytomegalovirus (CMV) & + & + & + \\
\hline Enterovirus & + & - & - \\
\hline $\begin{array}{l}\text { Human immunodeficiency virus } \\
\text { (HIV-1/2) }\end{array}$ & + & + & - \\
\hline Norovirus & + & - & - \\
\hline Parechovirus & $(+)$ & - & - \\
\hline Rotavirus & + & + & - \\
\hline Sapovirus & $(+)$ & - & - \\
\hline
\end{tabular}

+ , existing risk; (+), low risk; -, no risk

a There is an ongoing debate whether these intestinal protozoa have pathogenic potential or should rather be seen as simple commensals of the gastrointestinal tract $[29,30]$.

which enable them to be selected out of the normal gastrointestinal flora, while other bacteria without such characteristics cannot be distinguished from apathogenic gut bacteria by culture methods alone (see below). Stool culture has important advantages, such as testing of isolated pathogens for antimicrobial susceptibility. As the successful antibiotic treatment of many bacterial intestinal infections requires knowledge of local resistance patterns (e.g. extent of fluoroquinolone-resistant Campylobacter strains), stool culture techniques remain mandatory to guide therapeutic interventions. However, these tests are laborious and require experienced personnel, and typically take 48-72 hours to obtain first results. Hence, other, more rapid diagnostic tests (RDTs) have been developed for some pathogens. For Campylobacter spp., for example, EIAs detecting a specific antigen in the stool proved to be a sensitive alternative to stool culture with results available within a few hours $[32,99]$. However, there is no international consensus on 
immunological assays for detection of Campylobacter spp. and no globally validated and standardised approach, so that these tools should not replace the selective stool culture [100]. PCR assays, characterised by high sensitivity and specificity, have been developed for most of the aforementioned bacteria. Thus far, however, integration into clinical routine testing is still limited. Important drawbacks are the high cost, the need for sophisticated laboratory equipment and well-trained laboratory technicians. Moreover, PCR cannot distinguish between dead or alive bacteria and does not allow testing for antimicrobial susceptibility. However, newly developed multiplex PCR assays are increasingly being evaluated as fast screening tests for early detection of various important enteric pathogens. Besides PCR, novel molecular diagnostics are currently being developed and validated for many bacterial and viral pathogens, e.g. loopmediated isothermal amplification (LAMP) assays. Results obtained thus far are promising $[101,102]$, but it remains to be elucidated whether such nucleic acid amplification tests can be employed on a larger scale in resource-constrained settings in the tropics.

\section{Salmonella enterica ser. Typhi/Paratyphi}

Diagnosis of enteric fever is challenging and often delayed or not performed due to the unavailability of the most sensitive techniques in areas of high endemicity [103]. In these settings, the Widal test (measuring an increasing $S$. Typhi-specific antibody titer over the course of 10 days in patient serum samples) is often the only available test, despite its poor diagnostic performance. Contrary to non-typhoidal salmonellosis, stool culture is not sufficiently sensitive to diagnose infection due to $S$. Typhi/S. Paratyphi. Culturing blood and bone marrow is more sensitive, but bone marrow aspiration is only rarely performed in tropical areas due to a lack of adequately equipped hospitals and laboratories [104]. Blood cultures should be obtained during the first week of disease to achieve adequate sensitivity [45]. Serotyping of isolates (e.g. by agglutination of $\mathrm{Vi}$ antigen or rapid detection of various antigens or IgM antibodies by different EIA kits) is helpful for a timely diagnosis, but lacks sensitivity and specificity [46]. PCR assays have been developed for different antigens of invasive S. enterica serovars, but still need further development and validation before they can be more widely recommended [105].

\section{Clostridium difficile}

C. difficile can be found as part of the physiological intestinal flora, but toxin-producing strains may cause severe diarrhoea, which is most frequently seen in hospitalised patients who recently received antibiotic treatment [33]. A selective stool culture (toxigenic culture, performed on a selective agar medium or after ethanol shock pretreatment) followed by tests for toxin production remains the diagnostic 'gold' standard for $C$. difficile [33] and is particularly useful when the quantity of toxins in stool samples is small [34]. A laborious and technically difficult cell culture cytotoxicity assay is still regarded as an alternative reference standard, but is seldom performed in most microbiological laboratories. More recently developed PCR assays targeting a toxin-encoding gene are currently discussed as an alternative method for early diagnosis of $C$. difficile infection. Such molecular methods allow a more precise characterisation of isolated C. difficile strains, e.g. ribotype differentiation $[35,106,107]$. Sensitivity and specificity of PCR have been reported to vary between $85 \%$ and $100 \%$ [108]. However, various molecular assays exist which are not yet fully standardised, and the diagnostic performance of commercially available kits may differ considerably from inhouse molecular testing methods in use at different laboratories. Of note, PCR can only prove the presence of the toxin-encoding gene, but cannot distinguish between asymptomatic carriage and acute infection.

In clinical practice, an easily applicable two-step approach is recommended for rapid and reasonably sensitive diagnosis of $C$. difficile [109]. Firstly, a screening test for $C$. difficile-associated glutamate dehydrogenase (GDH) should be performed to indicate the bacterium's presence in a stool sample. If positive, it should be followed by a test for toxin production (e.g. toxin A/B EIA). This procedure does not require an extensively equipped laboratory and generates accurate results within a few hours. However, the sensitivity and specificity of this two-step approach are limited, and hence toxigenic culture and PCR testing should always be performed when there is a high clinical suspicion despite negative test results [110].

\section{Pathogenic Escherichia coli strains}

Diagnosis of pathogenic E. coli is challenging, as these bacteria constitute an important part of the physiological intestinal flora and only some strains have diarrhoeagenic potential [41]. There are at least six groups of pathogenic E. coli strains, namely (i) diffusely adherent (DAEC); (ii) enteroaggregrative (EAEC); (iii) enterohaemorrhagic (EHEC, including STEC = shiga toxin-producing E. coli); (iv) enteroinvasive (EIEC); (v) enteropathogenic (EPEC); and (vi) enterotoxigenic E. coli (ETEC). Pathogenic E. coli strains that carry simultaneously virulence factors from different pathotypes may cause severe clinical outbreaks. In mid-2011 in Germany, for example, the E. coli strain O104: H4 (an EAEC capable of EHEC/STEC-specific shiga toxin production) caused 2,987 cases of acute, often severe gastroenteritis and 855 cases of haemolytic-uraemic syndrome which led to 53 deaths [111].

While diagnostic procedures are poorly standardised for the pathotypes DAEC and EAEC, molecular 
Table 2 Diagnostic tests for important bacterial pathogens that may cause persistent digestive disorders

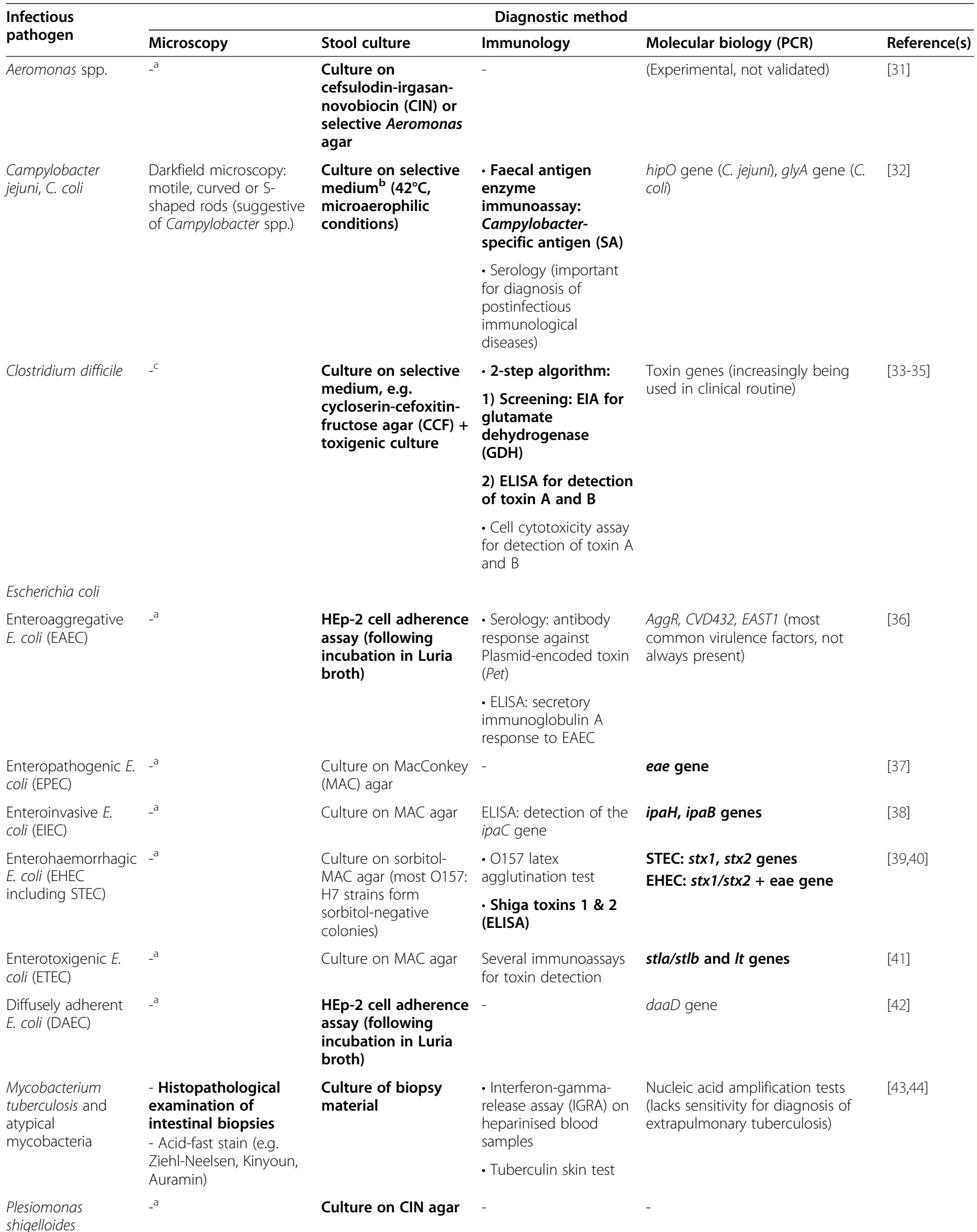


Table 2 Diagnostic tests for important bacterial pathogens that may cause persistent digestive disorders (Continued)

\begin{tabular}{|c|c|c|c|c|c|}
\hline \multirow{2}{*}{$\begin{array}{l}\text { Infectious } \\
\text { pathogen }\end{array}$} & \multicolumn{5}{|c|}{ Diagnostic method } \\
\hline & Microscopy & Stool culture & Immunology & Molecular biology (PCR) & Reference(s) \\
\hline $\begin{array}{l}\text { Salmonella enterica } \\
\text { (typhoidal and non- } \\
\text { typhoidal serovars) }\end{array}$ & $-{ }^{a}$ & $\begin{array}{l}\text { - Culture }{ }^{\mathbf{d}} \text { from } \\
\text { blood and/or bone } \\
\text { marrow (enteric fever) } \\
\text { - Culture from stool } \\
\text { or duodenal aspirate } \\
\text { (typhoidal and non- } \\
\text { typhoidal } \\
\text { salmonellosis) }\end{array}$ & $\begin{array}{l}\text { - Serotyping of isolates } \\
\text { (Vi antigen) } \\
\text { - ELISA: detection of } \\
\text { S. typhi antigens (blood) } \\
\text { - Widal agglutination } \\
\text { test (commonly used } \\
\text { in Africa) }\end{array}$ & $\begin{array}{l}\text { (Mainly for research } \\
\text { purpose) }\end{array}$ & {$[45-47]$} \\
\hline $\begin{array}{l}\text { Shigella dysenteriae, } \\
\text { S. flexneri, S. boydii, } \\
\text { S. sonnei }\end{array}$ & $-{ }^{a}$ & $\begin{array}{l}\text { Culture on MAC, } \\
\text { XLD, HE or Leifson } \\
\text { agar }\end{array}$ & $\begin{array}{l}\text { Agglutination tests to } \\
\text { detect serogroup and } \\
\text { serotype }\end{array}$ & ipaH, ip/ genes & {$[48]$} \\
\hline Tropheryma whipplei & $\begin{array}{l}\text { Histopathological } \\
\text { examination of PAS- } \\
\text { stained intestinal } \\
\text { biopsies: sickleform } \\
\text { particle-containing } \\
\text { cells }\end{array}$ & $\begin{array}{l}\text { (Only in highly } \\
\text { specialised } \\
\text { laboratories) }\end{array}$ & $\begin{array}{l}\text { Immunohistochemistry } \\
\text { on PAS-positive biopsy } \\
\text { material }\end{array}$ & whip1, whip2 genes & {$[49]$} \\
\hline Vibrio spp. & $\begin{array}{l}\text { Darkfield microscopy: } \\
\text { comma-shaped, motile } \\
\text { bacteria (highly } \\
\text { suggestive of Vibrio spp.) }\end{array}$ & $\begin{array}{l}\text { Culture on TCBS } \\
\text { agar }\end{array}$ & - & $\begin{array}{l}\text { PCR for species } \\
\text { differentiation } \\
\text { (V. cholerae, V. parahaemolyticus, } \\
\text { V. vulnificus) }\end{array}$ & {$[50,51]$} \\
\hline $\begin{array}{l}\text { Yersinia } \\
\text { enterocolitica, } \\
\text { Y. pseudotuberculosis }\end{array}$ & $-{ }^{a}$ & Culture on CIN agar & $\begin{array}{l}\text { Serology (important for } \\
\text { diagnosis of } \\
\text { postinfectious } \\
\text { immunological } \\
\text { diseases) }\end{array}$ & $\begin{array}{l}\text { PCR (reference } \\
\text { laboratories and research purposes) }\end{array}$ & {$[52]$} \\
\hline
\end{tabular}

The laboratory techniques are divided into different categories and recommended tests for each pathogen are highlighted.

${ }^{a}$ Gram staining of stool samples can be useful to evaluate the presence of leucocytes, but is not helpful to differentiate between pathogenic bacteria and apathogenic microbial flora.

${ }^{\mathrm{b}}$ Commonly employed selective media for detection of Campylobacter spp. include charcoal-cefoperazone-deoxycholate agar, Campylobacter blood agar plate, and cefoperazone-vancomycin-amphotericin agar [53].

' Detection of $C$. difficile in the Gram stain is not adequate to differentiate between clinical infection and simple colonisation with $C$. difficile [54].

${ }^{d}$ Commonly employed selective media for growth of S. enterica are MAC, XLD, HE, Leifson agar or other chromogenic media.

biological testing has revolutionized the diagnostic algorithms for the other diarrhoeagenic E. coli. Modern multiplex PCR assays targeting unique genes of EHEC/ STEC, EIEC, EPEC and ETEC allow a rapid molecular characterisation of these pathogenic strains. Hence, multiplex PCR assays have become the test of choice with excellent sensitivity and specificity (>99\%) [42]. Indeed, these tests have overcome important drawbacks of the classical stool culture, which often detects only some important strains (e.g. in the case of EHEC the O157:H7 strain on Sorbitol-MacConkey agar), but misses others that lack characteristic biochemical properties [39]. However, the integration of such multiplex PCR assays into routine testing of clinical samples remains restricted to well-equipped laboratories, and hence, these molecular techniques are only rarely available in endemic settings in the tropics.

\section{Mycobacterium tuberculosis and atypical mycobacteria (e.g. M. avium)}

Gastrointestinal tuberculosis is the sixth most common manifestation of extrapulmonary tuberculosis and causes considerable morbidity, including persistent diarrhoea and abdominal pain [112]. Atypical mycobacteria (synonymous: mycobacteria other than tuberculosis, MOTT), particularly M. avium, are an important cause of long-lasting diarrhoea and gastrointestinal complaints in HIV-infected individuals. Accurate diagnosis is difficult and relies on in-depth analysis of intestinal biopsy specimens by histopathological examination, microscopy after acid-fast staining (e.g. Ziehl-Neelsen, Auramin or Kinyoun techniques) and culture on selective media suitable for mycobacteria. Unless performed using oil immersion, histopathology often fails to distinguish between gastrointestinal tuberculosis and other granulomatous disorders, such as Crohn's disease [113,114]. An important drawback when culturing mycobacteria is their slow growth; it might take up to six weeks until cultures become positive. However, culture is the most sensitive technique and remains the diagnostic 'gold' standard [115]. Different molecular biological assays have been developed for various mycobacteria, but lack sensitivity for extrapulmonary tuberculosis and have not yet been validated for gastrointestinal tuberculosis [43]. 
Table 3 Diagnostic tests for important intestinal protozoa that may cause persistent digestive disorders

\begin{tabular}{|c|c|c|c|c|c|}
\hline \multirow{2}{*}{$\begin{array}{l}\text { Infectious } \\
\text { pathogen }\end{array}$} & \multicolumn{5}{|c|}{ Diagnostic method } \\
\hline & Microscopy & Stool culture & Immunology & $\begin{array}{l}\text { Molecular } \\
\text { biology (PCR) }\end{array}$ & Reference(s) \\
\hline \multirow[t]{4}{*}{ Balantidium coli } & Stool microscopy & \multirow[t]{4}{*}{-} & \multirow[t]{4}{*}{-} & \multirow[t]{4}{*}{-} & \multirow[t]{4}{*}[55]{} \\
\hline & $\begin{array}{l}\text { - Wet mount smears } \\
\text { (unstained or iodine } \\
\text { stain) }\end{array}$ & & & & \\
\hline & $\begin{array}{l}\text { - Concentration } \\
\text { techniques (e.g. formalin- } \\
\text { ether) }\end{array}$ & & & & \\
\hline & $\begin{array}{l}\text { Permanent stains (e.g. } \\
\text { with iron hematoxylin) }\end{array}$ & & & & \\
\hline \multirow[t]{3}{*}{ Blastocystis hominis } & Stool microscopy & \multirow{3}{*}{$\begin{array}{l}\text { Stool culture on selective } \\
\text { liquid media (no routine } \\
\text { procedure, but beneficial in } \\
\text { microscopically uncertain } \\
\text { cases) }\end{array}$} & \multirow[t]{3}{*}{ (No routine procedure) } & \multirow{3}{*}{$\begin{array}{l}\text { (PCR mainly } \\
\text { applied in } \\
\text { research } \\
\text { settings) }\end{array}$} & \multirow[t]{3}{*}[56-58]{} \\
\hline & $\begin{array}{l}\text { Wet mount smears } \\
\text { (unstained or iodine } \\
\text { stain) }\end{array}$ & & & & \\
\hline & $\begin{array}{l}\text { Permanent stains (e.g. } \\
\text { with trichrome, iron } \\
\text { hematoxylin, Giemsa) }\end{array}$ & & & & \\
\hline \multirow[t]{3}{*}{$\begin{array}{l}\text { Cryptosporidium } \\
\text { spp. }\end{array}$} & $\begin{array}{l}\text { Stool microscopy } \\
\text { - Wet mount smears }\end{array}$ & \multirow[t]{3}{*}{ (No routine procedure) } & $\begin{array}{l}\text { - ELISA: faecal antigen } \\
\text { detection }\end{array}$ & \multirow{3}{*}{$\begin{array}{l}\text { PCR } \text { (in } \\
\text { reference } \\
\text { laboratories and } \\
\text { for species } \\
\text { differentiation) }\end{array}$} & \multirow[t]{3}{*}[59-62]{} \\
\hline & & & \multirow[t]{2}{*}{ - Fluorescence microscopy } & & \\
\hline & $\begin{array}{l}\text { - Various staining } \\
\text { techniques, especially } \\
\text { acid-fast stains (e.g. } \\
\text { Kinyoun, modified Ziehl- } \\
\text { Neelsen) }\end{array}$ & & & & \\
\hline \multirow{4}{*}{$\begin{array}{l}\text { Cyclospora } \\
\text { cayetanensis }\end{array}$} & Stool microscopy & \multirow[t]{4}{*}{ (No routine procedure) } & \multirow[t]{4}{*}{-} & \multirow{4}{*}{$\begin{array}{l}\text { PCR (in } \\
\text { reference } \\
\text { laboratories) }\end{array}$} & \multirow[t]{4}{*}{ [63] } \\
\hline & $\begin{array}{l}\text { - Wet mount smears } \\
\text { (light or epifluorescence } \\
\text { microscopy) }\end{array}$ & & & & \\
\hline & $\begin{array}{l}\text { - Concentration } \\
\text { techniques (e.g. formalin- } \\
\text { ether) }\end{array}$ & & & & \\
\hline & $\begin{array}{l}\text { - Acid-fast stains (oocysts } \\
\text { are variably acid-fast) }\end{array}$ & & & & \\
\hline $\begin{array}{l}\text { Dientamoeba } \\
\text { fragilis }\end{array}$ & $\begin{array}{l}\text { Stool microscopy on } \\
\text { stained smears (e.g. iron- } \\
\text { hematoxylin, chlorazol } \\
\text { black dye stain) }\end{array}$ & (No routine procedure) & - & $\begin{array}{l}\text { PCR } \text { (in } \\
\text { reference } \\
\text { laboratories) on } \\
\text { unpreserved } \\
\text { stool samples }\end{array}$ & {$[64,65]$} \\
\hline \multirow{4}{*}{$\begin{array}{l}\text { Entamoeba } \\
\text { histolytica }\end{array}$} & Stool microscopy & \multirow[t]{4}{*}{ (No routine procedure) } & \multirow{2}{*}{$\begin{array}{l}\text { - ELISA: faecal antigen } \\
\text { detection able to distinguish } \\
\text { between E. histolytica and } \\
\text { E. dispar/moshkovskii (stool) }\end{array}$} & \multirow{4}{*}{$\begin{array}{l}\text { PCR (in } \\
\text { reference } \\
\text { laboratories) }\end{array}$} & \multirow[t]{4}{*}[66-70]{} \\
\hline & $\begin{array}{l}\text { - Wet mount smears } \\
\text { (trophozoites) }\end{array}$ & & & & \\
\hline & $\begin{array}{l}\text { - Formalin-ether } \\
\text { concentration (cysts) }\end{array}$ & & \multirow{2}{*}{$\begin{array}{l}\text { - Serological antibody } \\
\text { detection tests (blood samples) }\end{array}$} & & \\
\hline & - Permanent stains & & & & \\
\hline \multirow{2}{*}{$\begin{array}{l}\text { Giardia intestinalis } \\
\text { (syn.: G. lamblia } \\
\text { and G. duodenalis) }\end{array}$} & Stool microscopy & \multirow[t]{2}{*}{ (No routine procedure) } & \multirow{2}{*}{$\begin{array}{l}\text { - ELISA: faecal antigen } \\
\text { detection }\end{array}$} & \multirow{2}{*}{$\begin{array}{l}\text { PCR (in } \\
\text { reference } \\
\text { laboratories) }\end{array}$} & \multirow[t]{2}{*}[60,71]{} \\
\hline & $\begin{array}{l}\text { - Wet mount smears } \\
\text { (trophozoites) }\end{array}$ & & & & \\
\hline
\end{tabular}

- Formalin-ether concentration (cysts)

- Permanent stains 
Table 3 Diagnostic tests for important intestinal protozoa that may cause persistent digestive disorders (Continued)

\begin{tabular}{|c|c|c|c|c|c|}
\hline \multirow{2}{*}{$\begin{array}{l}\text { Infectious } \\
\text { pathogen }\end{array}$} & \multicolumn{5}{|c|}{ Diagnostic method } \\
\hline & Microscopy & Stool culture & Immunology & $\begin{array}{l}\text { Molecular } \\
\text { biology (PCR) }\end{array}$ & Reference(s) \\
\hline \multirow{4}{*}{$\begin{array}{l}\text { Isospora belli (syn.: } \\
\text { Cystoisospora belli) }\end{array}$} & Stool microscopy & \multirow[t]{4}{*}{-} & \multirow[t]{4}{*}{-} & \multirow{4}{*}{$\begin{array}{l}\text { PCR (in } \\
\text { reference } \\
\text { laboratories) }\end{array}$} & \multirow[t]{4}{*}[60,72,73]{} \\
\hline & - Wet mount smears & & & & \\
\hline & $\begin{array}{l}\text { - Concentration techniques } \\
\text { (e.g. formalin-ether) }\end{array}$ & & & & \\
\hline & - Acid-fast stains & & & & \\
\hline \multirow{2}{*}{$\begin{array}{l}\text { Species of } \\
\text { microsporidia } \\
\text { (Enterocytozoon } \\
\text { bieneusi, } \\
\text { Encephalitozoon } \\
\text { spp.) }\end{array}$} & $\begin{array}{l}\text { - Transmission electron } \\
\text { microscopy (gold standard, } \\
\text { but not feasible as routine } \\
\text { test) }\end{array}$ & \multirow[t]{2}{*}{-} & \multirow[t]{2}{*}{$\begin{array}{l}\text { Serology: anti-microsporidial } \\
\text { antibodies (indirect } \\
\text { immunofluorescence assay) }\end{array}$} & \multirow[t]{2}{*}{$\begin{array}{l}\text { PCR (in } \\
\text { reference } \\
\text { laboratories) }\end{array}$} & \multirow[t]{2}{*}[60,74-76]{} \\
\hline & $\begin{array}{l}\text { - Light microscopy (e.g. } \\
\text { Uvitex B, Chromotrope R or } \\
\text { Calcofluor White stain) }\end{array}$ & & & & \\
\hline
\end{tabular}

The laboratory techniques are divided into different categories and recommended tests for each pathogen are highlighted.

\section{Tropheryma whipplei}

Whipple's disease due to infection with T. whipplei is a rare disease characterised by chronic diarrhoea, wasting, abdominal pain, arthralgia and various other symptoms associated with organ involvement (e.g. encephalitis and endocarditis) [49]. The infectious agent was not identified until 1961 and many epidemiological and biological features still need to be elucidated [116]. Only highly specialised laboratories are able to grow T. whipplei on human fibroblast cells $[117,118]$. The development of a PCR assay targeting the genes whip1 and whip2 has been a major step forward and is nowadays the test of choice, especially in symptomatic patients without typical histopathological findings in intestinal biopsies (sickleform particle-containing cells on periodic acidSchiff (PAS-)stained biopsy specimens) [49].

Parasitic pathogens: intestinal protozoa Balantidium coli, Blastocystis hominis, Cryptosporidium spp., Cyclospora cayetanensis, Dientamoeba fragilis, Entamoeba histolytica, Giardia intestinalis (syn.: G. Iamblia and G. duodenalis), Isospora belli (syn.: Cystoisospora belli), species of microsporidia

The three main techniques for the diagnosis of human intestinal protozoan infections include (i) light microscopy; (ii) antigen detection (EIAs); and (iii) PCR assays. Since the first description of parasitic intestinal protozoa in human stools, documented by the Dutch microscopist Antony van Leeuwenhoek in 1681 [119], microscopic detection of protozoan cysts and trophozoites has been the most widely used diagnostic approach. On fresh stool samples, direct microscopy is performed by mixing a small amount of faeces with a physiological $0.9 \%$ sodium chloride $(\mathrm{NaCl})$ solution. To increase sensitivity, various stool concentration techniques have been developed, making use of either sedimentation or flotation with a formalin-ether concentration technique being the most widely used method in medical laboratories [120,121]. However, the formalin-ether concentration technique lacks sensitivity for several intestinal protozoan species as well as many helminths (described below), and hence there is a pressing need for new and more sensitive microscopic techniques (e.g. FLOTAC) [122] and non-microscopic diagnostics. Staining techniques can be helpful for microscopic parasite identification and might further improve the diagnostic accuracy. Indeed, some intestinal protozoan species require staining of the stool sample to be identified on microscopic examination. For example, acid-fast stains allow detection of Cryptosporidium spp., while species of microsporidia are best seen when using an Uvitex B or Calcofluor White stain. Still, correct identification of intestinal protozoan pathogens is challenging even for experienced laboratory technicians and for some species even impossible (e.g. E. histolytica based on cysts morphology). For Cryptosporidium spp., E. histolytica and G. intestinalis, sensitive EIAs detecting speciesspecific antigens in faecal samples have been developed, some of which are highly sensitive and complement microscopic stool examination in many clinical laboratories $[123,124]$. Especially for the diagnosis of E. histolytica, species differentiation based on alternative procedures is compulsory, since microscopy cannot readily distinguish between $E$. histolytica and the non-pathogenic $E$. dispar $[66,125,126]$. Of note, not all commercially available EIA antigen detection kits are E. histolytica-specific and some lack sensitivity, in particular if faecal samples have been stored for several days $[67,127]$. Over the past several years, highly sensitive PCR assays have been developed and standardised for many intestinal protozoan species. 
Table 4 Diagnostic tests for important helminths that may cause persistent digestive disorders

\begin{tabular}{|c|c|c|c|c|c|}
\hline \multirow[t]{2}{*}{ Infectious pathogen } & \multicolumn{5}{|c|}{ Diagnostic method } \\
\hline & Microscopy & Stool culture & Immunology & Molecular biology (PCR) & Reference(s) \\
\hline \multicolumn{6}{|l|}{ Cestodes } \\
\hline \multirow[t]{4}{*}{ Diphyllobothrium latum } & $\begin{array}{l}\text { Stool microscopy: } \\
\text { identification of eggs or } \\
\text { proglottids }\end{array}$ & \multirow[t]{4}{*}{-} & \multirow[t]{4}{*}{-} & \multirow[t]{4}{*}{$\begin{array}{l}\text { PCR and sequencing for species } \\
\text { differentiation (for } \\
\text { epidemiological purpose) }\end{array}$} & \multirow[t]{4}{*}[77,78]{} \\
\hline & - Wet preparation & & & & \\
\hline & $\begin{array}{l}\text { - Ethyl-acetate or } \\
\text { formalin-ether-based } \\
\text { concentration techniques }\end{array}$ & & & & \\
\hline & $\begin{array}{l}\text { Sedimentation } \\
\text { techniques }\end{array}$ & & & & \\
\hline \multirow[t]{5}{*}{ Hymenolepis spp. } & Stool microscopy & \multirow[t]{5}{*}{-} & \multirow[t]{5}{*}{-} & \multirow{5}{*}{$\begin{array}{l}P C R \text { in research settings (for } \\
\text { epidemiological purpose) }\end{array}$} & \multirow[t]{5}{*}[79]{} \\
\hline & - Kato-Katz method & & & & \\
\hline & $\begin{array}{l}\text { - Ethyl-acetate or } \\
\text { formalin-ether-based } \\
\text { concentration techniques }\end{array}$ & & & & \\
\hline & $\begin{array}{l}\text { - Sedimentation } \\
\text { techniques }\end{array}$ & & & & \\
\hline & • FLOTAC & & & & \\
\hline \multirow[t]{4}{*}{ Taenia spp. } & Stool microscopy & \multirow[t]{4}{*}{-} & - Coproantigen EIA & \multirow[t]{4}{*}{ PCR for species differentiation } & \multirow[t]{4}{*}[80]{} \\
\hline & - Perianal egg detection & & \multirow{3}{*}{$\begin{array}{l}\text { - Serology: detection } \\
\text { of specific circulating } \\
\text { antibodies against } \\
\text { T. solium }\end{array}$} & & \\
\hline & $\begin{array}{l}\text { - (Graham's test applying } \\
\text { adhesive tape) }\end{array}$ & & & & \\
\hline & $\begin{array}{l}\text { - Examination of tapeworms } \\
\text { from purges }\end{array}$ & & & & \\
\hline \multicolumn{6}{|l|}{ Nematodes } \\
\hline \multirow[t]{5}{*}{ Ascaris lumbricoides } & $\begin{array}{l}\text { Stool microscopy: egg } \\
\text { detection }\end{array}$ & \multirow[t]{5}{*}{-} & \multirow[t]{5}{*}{-} & \multirow[t]{5}{*}{$\begin{array}{l}\mathrm{PCR} \text { in research settings (for } \\
\text { epidemiological purpose) }\end{array}$} & \multirow[t]{5}{*}[81-83]{} \\
\hline & - Kato-Katz method & & & & \\
\hline & $\begin{array}{l}\text { - Ethyl-acetate or } \\
\text { formalin-ether-based } \\
\text { concentration techniques }\end{array}$ & & & & \\
\hline & $\begin{array}{l}\text { - Sedimentation } \\
\text { techniques }\end{array}$ & & & & \\
\hline & • FLOTAC & & & & \\
\hline \multirow[t]{4}{*}{ Capillaria philippinensis } & $\begin{array}{l}\text { Stool microscopy: egg } \\
\text { detection }\end{array}$ & \multirow[t]{4}{*}{-} & \multirow[t]{4}{*}{-} & \multirow[t]{4}{*}{-} & \multirow[t]{4}{*}[84,85]{} \\
\hline & $\begin{array}{l}\text { - Ethyl-acetate or } \\
\text { formalin-ether-based } \\
\text { concentration techniques }\end{array}$ & & & & \\
\hline & $\begin{array}{l}\text { Sedimentation } \\
\text { techniques }\end{array}$ & & & & \\
\hline & $\begin{array}{l}\text { - (Kato-Katz method: great } \\
\text { care is indicated to distinguish } \\
\text { between T. trichiura and } \\
\text { C. philippinensis eggs) }\end{array}$ & & & & \\
\hline \multirow{3}{*}{$\begin{array}{l}\text { Hookworms } \\
\text { (Ancylostoma } \\
\text { duodenale, Necator } \\
\text { americanus) }\end{array}$} & $\begin{array}{l}\text { Stool microscopy: egg } \\
\text { detection }\end{array}$ & \multirow{3}{*}{$\begin{array}{l}\text { Culture on Koga agar } \\
\text { and subsequent } \\
\text { microscopic } \\
\text { identification of } \\
\text { larvae }\end{array}$} & & \multirow{3}{*}{$\begin{array}{l}\text { PCR mainly applied in research } \\
\text { settings (for epidemiological } \\
\text { purpose) }\end{array}$} & {$[81-83]$} \\
\hline & - Kato-Katz method & & & & \\
\hline & $\begin{array}{l}\text { - Ethyl-acetate or formalin- } \\
\text { ether-based concentration } \\
\text { techniques }\end{array}$ & & & & \\
\hline
\end{tabular}

- Sedimentation techniques 
Table 4 Diagnostic tests for important helminths that may cause persistent digestive disorders (Continued)

\begin{tabular}{|c|c|c|c|c|c|}
\hline \multirow[t]{2}{*}{ Infectious pathogen } & \multicolumn{5}{|c|}{ Diagnostic method } \\
\hline & Microscopy & Stool culture & Immunology & Molecular biology (PCR) & Reference(s) \\
\hline \multirow[t]{3}{*}{ Strongyloides stercoralis } & \multirow{3}{*}{$\begin{array}{l}\text { - Stool: microscopy } \\
\text { following Baermann } \\
\text { funnel concentration } \\
\text { - Microscopy of sputum, } \\
\text { bronchoalveolar lavage, } \\
\text { duodenal aspirate, skin } \\
\text { biopsy }\end{array}$} & \multirow{3}{*}{$\begin{array}{l}\text { Culture on Koga } \\
\text { agar and } \\
\text { subsequent } \\
\text { microscopic } \\
\text { identification of } \\
\text { larvae }\end{array}$} & \multirow{2}{*}{$\begin{array}{l}\text { - ELISA tests } \\
\text { detecting serum } \\
\text { antibodies or faecal } \\
\text { antigens }\end{array}$} & \multirow{3}{*}{$\begin{array}{l}\text { PCR applied in research } \\
\text { settings (for epidemiological } \\
\text { purpose) and increasingly used } \\
\text { for individual patient } \\
\text { management }\end{array}$} & \multirow[t]{3}{*}[86,87]{} \\
\hline & & & & & \\
\hline & & & $\begin{array}{l}\text { - Indirect fluorescent } \\
\text { antibody test }\end{array}$ & & \\
\hline \multirow[t]{5}{*}{ Trichuris trichiura } & $\begin{array}{l}\text { Stool microscopy: egg } \\
\text { detection }\end{array}$ & \multirow[t]{5}{*}{-} & \multirow[t]{5}{*}{-} & \multirow[t]{6}{*}{-} & \multirow[t]{5}{*}[81,82]{} \\
\hline & - Kato-Katz method & & & & \\
\hline & $\begin{array}{l}\text { - Ethyl-acetate or } \\
\text { formalin-ether-based } \\
\text { concentration techniques }\end{array}$ & & & & \\
\hline & $\begin{array}{l}\text { - Sedimentation } \\
\text { techniques }\end{array}$ & & & & \\
\hline & • FLOTAC & & & & \\
\hline \multicolumn{5}{|l|}{ Trematodes } & \\
\hline \multirow[t]{6}{*}{ Intestinal flukes } & $\begin{array}{l}\text { Stool microscopy: egg } \\
\text { detection }\end{array}$ & \multirow[t]{6}{*}{-} & \multirow{6}{*}{$\begin{array}{l}\text { ELISA to detect } \\
\text { worm-specific } \\
\text { antibodies or } \\
\text { antigens in serum or } \\
\text { stool }\end{array}$} & \multirow{6}{*}{$\begin{array}{l}\text { PCR applied in research } \\
\text { settings (for epidemiological } \\
\text { purpose) }\end{array}$} & \multirow[t]{6}{*}[88]{} \\
\hline & - Kato-Katz method & & & & \\
\hline & $\begin{array}{l}\text { - Ethyl-acetate or } \\
\text { formalin-ether-based } \\
\text { concentration techniques }\end{array}$ & & & & \\
\hline & - Stoll's dilution & & & & \\
\hline & $\begin{array}{l}\text { - Sedimentation } \\
\text { techniques }\end{array}$ & & & & \\
\hline & • FLOTAC & & & & \\
\hline \multirow{8}{*}{$\begin{array}{l}\text { Intestinal blood flukes: } \\
\text { Schistosoma mansoni, } \\
\text { S. intercalatum, } \\
\text { S. japonicum, } \\
\text { S. mekongi }\end{array}$} & $\begin{array}{l}\text { Stool microscopy: egg } \\
\text { detection }\end{array}$ & \multirow[t]{8}{*}{-} & \multirow{3}{*}{$\begin{array}{l}\text { - ELISA to detect } \\
\text { serum antibodies or } \\
\text { worm-specific } \\
\text { antigens in serum or } \\
\text { urine }\end{array}$} & \multirow{8}{*}{$\begin{array}{l}\text { PCR applied in research } \\
\text { settings for epidemiological } \\
\text { purpose and increasingly used } \\
\text { for individual patient } \\
\text { management }\end{array}$} & \multirow[t]{8}{*}{ [89] } \\
\hline & - Kato-Katz method & & & & \\
\hline & \multirow{2}{*}{$\begin{array}{l}\text { - Ethyl-acetate or } \\
\text { formalin-ether-based } \\
\text { concentration techniques }\end{array}$} & & & & \\
\hline & & & \multirow{5}{*}{$\begin{array}{l}\text { - RDT to detect CCA } \\
\text { or CAA antigen in } \\
\text { serum or urine (for } \\
\text { S. mansoni) }\end{array}$} & & \\
\hline & - Stoll's dilution & & & & \\
\hline & $\begin{array}{l}\text { - Sedimentation } \\
\text { techniques }\end{array}$ & & & & \\
\hline & $\begin{array}{l}\text { - FLOTAC (first experiences } \\
\text { for S. mansoni) }\end{array}$ & & & & \\
\hline & $\begin{array}{l}\text { Miracidium-hatching test } \\
\text { from stool samples }\end{array}$ & & & & \\
\hline
\end{tabular}

The laboratory techniques are divided into different categories and recommended tests for each pathogen are highlighted.

Many of these assays (e.g. Entamoeba spp. differentiation by PCR) are currently being integrated into parasitological reference laboratories as an additional diagnostic tool to prove diagnosis in uncertain clinical cases $[59,128,129]$. Such molecular biological tools are of enormous importance to improve the correct species identification of many intestinal parasites, which are difficult to diagnose using conventional techniques $[60,74]$.
Parasitic pathogens: helminths

Ascaris lumbricoides, Capillaria philippinensis,

Diphyllobothrium spp., Hymenolepis spp., hookworm

(Ancylostoma duodenale and Necator americanus), Taenia

spp., Trichuris trichiura, intestinal flukes

Identification of helminth eggs on microscopic stool examination is the reference test for most intestinal helminth species. In hospitals and microbiological laboratories, 
direct stool examination after prior concentration (e.g. by formalin-ether concentration technique) is most commonly employed, while the Kato-Katz thick smear technique is widely used in epidemiological studies and anthelminthic drug efficacy evaluations in endemic regions [81,130-132]. Direct microscopic examination is a cheap methodology, the microscope slides can rapidly be prepared for examination, and there is no need for sophisticated laboratory equipment. The eggs of most helminth species parasitising humans can easily be distinguished by a trained laboratory technician (see Figure 1 for eight selected helminth eggs). Hence, microscopy remains the standard reference test for A. lumbricoides, T. trichiura, hookworm, Capillaria philippinensis, Diphyllobothrium spp., Hymenolepis spp., Taenia spp. and blood flukes (Schistosoma spp.) [82,88,133,134]. However, microscopy is prone to a number of shortcomings. Firstly, microscopy is not very sensitive and especially infections of light intensity can be missed when only a single stool sample is analysed [131,135]. Multiple stool sampling, ideally over several consecutive days, increases the sensitivity [136], as well as the use of different concentration techniques, which are based on sedimentation (e.g. formalin-ether concentration technique), flotation or a combination of both (e.g. McMaster technique and FLOTAC) [135,137-139].
However, these techniques often require access to the power grid, a centrifuge and different chemical reagents, which are not always available in tropical settings. Moreover, the diagnostic sensitivity for different helminth species often varies considerably, and no currently available concentration technique is able to concurrently detect intestinal protozoa and helminths with the same diagnostic accuracy $[122,140]$.

Secondly, microscopy results heavily depend on the quality of the slide preparation and on the experience of the laboratory technician reading the slides. Thirdly, the eggs of some helminth species such as the two hookworm species A. duodenale and N. americanus are virtually indistinguishable by microscopy. Fourthly, the nematode Strongyloides stercoralis can rarely be found when using the aforementioned microscopy techniques, because its larvae already hatch in the intestine and, hence, the eggs are not passed in the faeces [141]. Despite all these constraints, microscopy is an invaluable tool for diagnostic medical parasitology.

New molecular techniques, especially PCR assays, still need to be validated and further developed in different settings. Disadvantages of current PCR tests are their high costs, risk of contamination, the need for hightechnology laboratory equipment and constant electric

Table 5 Diagnostic tests for important viral pathogens that may cause persistent digestive disorders

\begin{tabular}{|c|c|c|c|c|c|}
\hline \multirow[t]{2}{*}{ Infectious pathogen } & \multicolumn{5}{|c|}{ Diagnostic method } \\
\hline & Electron microscopy & Cell culture & Immunology & $\begin{array}{l}\text { Molecular } \\
\text { biology (PCR) }\end{array}$ & Reference(s) \\
\hline \multicolumn{6}{|l|}{ Viruses } \\
\hline Adenovirus & $\begin{array}{l}\text { Low sensitivity } \\
\left(>10^{6} \text { viral particles } / \mathrm{ml}\right)\end{array}$ & A549-, HEp-2-, HEK-cells & $\begin{array}{l}\text { Antigen detection in faecal samples } \\
\text { (ELISA, immunochromatography) }\end{array}$ & PCR & {$[90]$} \\
\hline Astrovirus & $\begin{array}{l}\text { Low sensitivity } \\
\left(>10^{6} \text { viral particles } / \mathrm{ml}\right)\end{array}$ & CaCO-2-, LLC-MK2-cells & Antigen detection (ELISA) & RT-PCR & [91] \\
\hline Bocavirus & - & - & - & PCR & [92] \\
\hline Coronavirus & - & - & - & RT-PCR & [93] \\
\hline \multirow[t]{2}{*}{ Cytomegalovirus (CMV) } & - & HFF-, MRC-5 cells & $\begin{array}{l}\text { - pp65 antigen detection } \\
\text { (immunofluorescence) }\end{array}$ & PCR & [91] \\
\hline & & $\begin{array}{l}\text { CMV-immediate early1- } \\
\text { pp72-antigen in HFF }\end{array}$ & $\begin{array}{l}\text { - (CMV-specific antibody } \\
\text { seroconversion) }\end{array}$ & & \\
\hline Enterovirus & - & MRC-5-, HEp-2-, Vero-cells & - & RT-PCR & [94] \\
\hline \multirow{2}{*}{$\begin{array}{l}\text { Human } \\
\text { immunodeficiency virus } \\
(\mathrm{HIV}-1 / 2)\end{array}$} & - & HUT-78-, CEM-MOLT4-cells & $\begin{array}{l}\text { - Immunoassay (e.g. 4th } \\
\text { generation) }\end{array}$ & RT-PCR & {$[95,96]$} \\
\hline & & & - Western Blot & & \\
\hline Norovirus & $\begin{array}{l}\text { Sensitivity } 10^{5}-10^{6} \\
\text { viral particles } / \mathrm{ml}\end{array}$ & - & $\begin{array}{l}\text { Antigen detection faecal samples } \\
(\text { EIA) }\end{array}$ & RT-PCR & [91] \\
\hline Parechovirus & - & - & - & RT-PCR & [97] \\
\hline Rotavirus & $\begin{array}{l}\text { Low sensitivity } \\
\left(>10^{6} \text { viral particles } / \mathrm{ml}\right)\end{array}$ & MA104-, CaCO-2-cells & $\begin{array}{l}\text { Antigen detection in faecal samples } \\
\text { (ELISA), rapid tests (ELISA, } \\
\text { immunochromatography) }\end{array}$ & RT-PCR & [91] \\
\hline Sapovirus & - & - & - & RT-PCR & [91] \\
\hline
\end{tabular}

The laboratory techniques are divided into different categories and recommended tests for each pathogen are highlighted. RT-PCR, reverse transcriptase-polymerase chain reaction. 

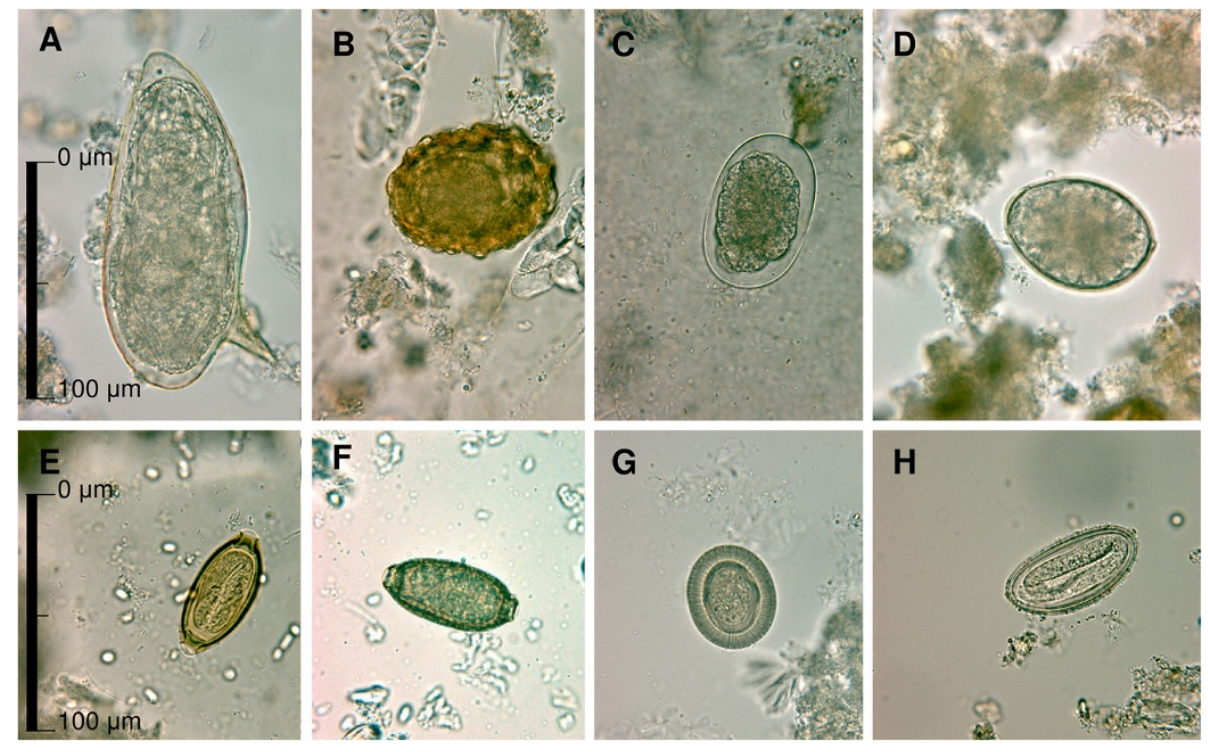

Figure 1 Morphological features of selected intestinal helminth eggs diagnosed using the formalin-ether concentration technique and standard light microscopy: A, Schistosoma mansoni; B, Ascaris lumbricoides; C, hookworm; D, Diphyllobothrium latum; E, Trichuris trichiura; F, Capillaria spp..; G, Taenia spp.; H, Enterobius vermicularis.

power supply which render their use for routine testing in many developing countries impossible. Indeed, PCR is seldom available in the most affected regions, and its results often do not guide clinicians' decisions, as empiric treatment with albendazole and mebendazole is commonly employed and effective against many helminth species in endemic areas [7]. Due to the variety of intestinal parasites causing digestive disorders, a multiplex real-time PCR targeting a host of various pathogens is much more desirable than individual PCR assays for each parasite, and such multiplex PCRs have been successfully developed and are increasingly used in reference laboratories in industrialised countries $[59,83,142]$. However, even these multiplex PCRs can only diagnose a defined host of targeted pathogens, while microscopy may sometimes detect unexpected pathogens that would have been missed by other diagnostic methods.

\section{Strongyloides stercoralis}

The diagnosis of S. stercoralis in human stool samples requires special, often laborious concentration techniques. Most commonly employed are the Baermann funnel and the Koga agar plate [143]. The Baermann method is a concentration technique based on the nematode's hydrophily and thermophily. It provides results within a few hours and is the technique of choice according to the World Gastroenterology Organization [144], but there is some debate whether it is as sensitive as agar plate cultures [86]. Derived from classical charcoal culture assays and its sequel, the so-called Harada-Mori culture, Koga and colleagues developed a special agar plate to detect $S$. stercoralis and hookworm larvae [145]. The agar plates are stored for 48 hours in a humid chamber and the traces of the helminths can then be seen on the agar and the larvae can easily be collected for microscopic species identification. In contrast to many other helminth infections, where exact species identification often is not necessarily required and clinical symptoms are mild, the recognition of strongyloidiasis and initiation of an effective treatment with ivermectin is essential to prevent potentially lifethreatening events due to its ability to cause disseminated hyperinfection in the immunosuppressed population $[141,146]$. Hence, the aforementioned laborious techniques seem to be justified and a combination of the Baermann funnel and the Koga agar plate method may lead to the most accurate results.

Recently, different PCR assays targeting the helminth's $18 \mathrm{~S}$ rRNA [87] or 28S rRNA [147] subunit have been developed. First results are promising, but still need further validation in endemic settings.

\section{Schistosoma mansoni, S. mekongi, S. intercalatum and S. japonicum}

The microscopic detection of blood fluke eggs in stool specimens still remains the cornerstone of the laboratory diagnosis of intestinal schistosomiasis, as the specificity is high and the costs of equipment are relatively low. However, the sensitivity fluctuates, depending on infection stage and intensity [148]. Hence, concentration methods like an ether-concentration, the Kato-Katz thick smear or the recently developed FLOTAC technique are important tools to increase sensitivity [89]. 
Moreover, examination of multiple (preferably at least three) stool samples collected on consecutive days is recommended $[136,149]$. In contrast to other helminth infections, immunological RDTs have been developed for detection of intestinal (S. mansoni) and urogenital schistosomiasis (S. haematobium). Worm-gut associated glycoproteins, namely circulating cathodic antigen (CCA) and circulating anodic antigen (CAA), can be detected in the serum and the urine of S. mansoniinfected individuals using genus-specific monoclonal antibodies [150,151]. Immunochromatographic point-ofcare (POC) dipstick or cassette tests for rapid diagnosis of $S$. mansoni via CCA detection in the urine are currently being validated in different epidemiological settings and will potentially become a valuable tool for non-microscopic diagnosis of schistosomiasis in epidemiological studies and clinical practice. Recent studies suggest that the diagnostic accuracy of a single POC-CCA test is considerably more sensitive than a single Kato-Katz thick smear and that a concurrent $S$. haematobium infection does not influence the POC-CCA test results for $S$. mansoni diagnosis, which is an important observation due to the co-endemicity of both blood fluke infections in many tropical areas [152,153]. Hence, antigen RDT assays will likely find their way into clinical practice in the foreseeable future.

PCR assays have been developed and are more sensitive than conventional parasitological and serological methods, but presently, their use is restricted to specialised reference laboratories and research institutions outside endemic areas [154,155].

\section{Viruses}

Viral infections commonly cause acute gastroenteritis with the highest burden concentrated in tropical and subtropical regions of the world. Even though these pathogens mainly lead to short-lasting and self-limiting diarrhoeal diseases, they account for considerable morbidity and even mortality, particularly in children [156]. In general, viral infections rarely cause chronic intestinal diseases, but must not be forgotten as potential pathogens that may give rise to persistent diarrhoea and chronic abdominal pain, particularly in HIV-infected individuals or otherwise immunocompromised hosts.

Traditionally, diagnosis of viral gastroenteritis is based on virus isolation by cell culture, electron microscopy and rapid antigen tests (e.g. latex agglutination or EIAs) [157]. Introduction of molecular methods led to an exponential increase in detection rates and the role of difficult-to-culture pathogens became apparent. From a technical point of view, most rapid tests can be done at the bedside, whereas cell culture, electron microscopy and molecular-based methods require laboratories with sophisticated equipment, experienced staff and appropriate biosafety procedures. This certainly limits the use of the latter methods in resource-constrained settings. Data on sensitivity and specificity of diagnostic tools for virus identification in tropical settings are currently lacking.

\section{Adenovirus}

Currently, more than 53 types of adenovirus are recognised which can cause a variety of clinical entities, but gastroenteritis is predominantly caused by types 40 and 41 $[158,159]$. In infected individuals, viral particles are shed in high concentrations. In general, virus isolation followed by serotyping remains the 'gold' standard for the detection of all serotypes and is possible on different cell lines (Table 5). Importantly, 293-Graham cells should be used for stool samples as adenovirus species $\mathrm{F}$ (adenovirus types 40 and 41) can only be cultivated on this cell line. However, virus isolation is rather laborious and timeconsuming in the face of urgent requests for diagnosis. Electron microscopy is possible with high specificity, but low sensitivity. As an alternative method that is particularly useful for examination of stool samples, antigen detection assays using EIA or latex agglutination have been developed [160,161]. These assays are rapid, but displayed varying sensitivities and specificities in studies, and hence should be complemented by alternative methods. Molecular methods, in particular real-time PCR, have demonstrated superior performance over conventional methods and are now the cornerstone for diagnosis in most laboratories, but are seldom available in resource-constrained settings.

\section{Astrovirus}

Eight serotypes of astrovirus are known. In childhood, astrovirus infection with serotypes 1 and 2 predominate, whereas infection with the other serotypes occurs later in life ( $>4$ years). Prolonged diarrhoea has been associated with astrovirus serotype 3 [162]. In the immunocompetent host, viral shedding occurs for 14-70 hours but may be prolonged in immunosuppressed patients. Virus propagation of astrovirus on CaCO-2 or LLCMK2 cells remains restricted to expert laboratories and is not recommended for routine diagnostic use. Virus identification by electron microscopy is possible, but appearance of viral particles is not always clear. Recently developed antigen detection kits have proven their suitability and are now widely available for rapid diagnosis. However, sensitivity and specificity of rapid tests in comparison to reverse transcriptase (RT)-PCR have been reported to be comparably low [163]. Real-time RT-PCR is the most sensitive and specific method, but remains restricted to reference laboratories. 


\section{Bocavirus}

Four different species of human bocavirus (hBoV) have been described thus far [164]. The diagnosis of hBoV infection is almost exclusively based on molecular methods. hBoV has not been isolated by cell culture or in an animal model and rapid antigen tests are currently not available. Serology (e.g. using viral-like particles) has been described and can be used to complement diagnosis [165]. A variety of PCR and real-time PCR assays have been described. However, due to prolonged detection of viral DNA at low copy numbers, qualitative detection of hBoV DNA in gastrointestinal samples is not recommended. There are only few data available for hBoV species 2-4 and the relevance as a true human pathogen is still under debate [164].

\section{Calicivirus}

The family calicivirus comprises two human-pathogenic genera, the norovirus and sapovirus [166]. For both genera, virus isolation by cell culture is not possible. Electron microscopy is rather insensitive and rarely detects the viruses if there are fewer than $10^{6}$ viral particles $/ \mathrm{ml}$ of stool suspension.

Norovirus Antigen EIAs have been developed and are commercially available for rapid diagnosis. They proved to be a valuable tool especially in outbreaks, but their sensitivity is limited [167]. A recent study from Brazil reported a sensitivity of $87.9 \%$ upon use of a 3rd generation norovirus antigen detection kit [168]. More recently, real-time RT-PCR assays have been described and demonstrated excellent sensitivity and specificity [169]. In-house methods as well as commercial kits are widely available and routinely used.

Sapovirus Specific real-time RT-PCR assays have been developed, but there are no comprehensive data evaluating their diagnostic accuracy. However, there are no diagnostic alternatives because rapid antigen tests are not yet available.

\section{Coronavirus}

Five different human pathogenic coronaviruses are known which can cause respiratory and/or to a lesser extent gastrointestinal symptoms in humans. However, the relevance of coronavirus as a true human enteric pathogen is unclear $[93,170]$. Conventional virus isolation by cell culture can be done on human embryonal tracheal cells. Electron microscopy is possible for stool samples but displays rather low sensitivity. For coronavirus, antigen tests for stool samples are not available. Molecular methods, e.g. real-time RT-PCR assays are the method of choice for a reliable and rapid diagnosis. However, most in-house methods are restricted to reference laboratories, and hence are not commonly employed around the globe.

\section{Cytomegalovirus}

In particular immunosuppressed patients are at risk for cytomegalovirus (CMV) infection, which can affect various organ systems, including the gastrointestinal tract $[158,171]$. Serology represents the method of choice to differentiate primary from secondary infection. Organspecific diagnosis (e. g. CMV-associated gastrointestinal disease) requires tissue biopsy samples. In combination with histopathology, isolation of CMV by cell culture is recommended. Detection of CMV-DNA by molecular methods alone is not sufficient.

\section{Enterovirus}

Enteroviruses belong to the family picornaviridae and comprise enterovirus group A to D [172]. In general, enteroviruses can cause a broad spectrum of different clinical entities. Gastroenteritis caused by coxsackievirus A is mostly seen in children. Virus isolation is possible on a range of different cell lines (Table 5). Virus typing after isolation is traditionally accomplished by virus neutralisation. Of note, enteroviruses may be shed into the stool for prolonged time after clearance of acute infection, thus limiting the significance of such a finding. RTPCR methods are now widely available for the detection of viral genomes. However, sequence variation among the different enterovirus groups can lower the specificity and PCR-based assays should regularly be updated using latest sequence information. Serological methods for the detection of enterovirus-specific antibodies are exclusively available in reference laboratories and cannot be used for rapid diagnosis.

\section{Parechovirus}

Parechoviruses have gained recent interest, but their role in acute gastroenteritis and persistent diarrhoea has yet to be established [173,174]. At the time of writing, 16 parechoviruses types have been described. They now represent an own genus within the familiy picornaviridae and realtime RT-PCR is the method of choice for diagnosis in high-income settings [175].

\section{Human rotavirus}

Rotavirus infection alone is believed to account for 453,000 deaths annually in children younger than 5 years [5]. In most cases, infection causes acute diarrhoea and vomiting with viral particles being shed in high concentrations. Virus isolation is possible on MA104 or CaCO-2-cells but remains laborious and time-consuming. Antigen detection 
by EIA methods is the current standard procedure for the rapid diagnosis of rotavirus infection and widely available for diagnosis as well as surveillance. These assays are able to detect virus particles even if their concentration is below $10^{4}$ particles $/ \mathrm{ml}$ stool suspension. Molecular methods are also available [176,177].

\section{HIV-associated enteropathy}

HIV-associated enteropathy frequently occurs in HIVinfected individuals without access to antiretroviral therapy and is characterised by persistent diarrhoea, weight loss, anorexia, abdominal pain and dysphagia. HIVassociated enteropathy should be diagnosed by obtaining intestinal biopsies via endoscopy with subsequent histological and microbiological examination [178]. Antiretroviral treatment of the HIV infection usually also cures the enteropathy.

\section{Discussion}

Persistent digestive disorders are unspecific clinical complaints which are commonly reported by many patients around the world. Gastrointestinal or systemic infections are important causes of such disorders with a broad spectrum of possible pathogens involved, including bacteria, intestinal protozoa, helminths and viruses. Due to the wide range of infectious agents which are often difficult to diagnose, great efforts have to be made to reach satisfactory detection rates and to avoid overlooking of important pathogens. Such a diagnostic work-up should include bacterial stool cultures on different selective media (including MacConkey, sorbitol-MacConkey, Leifson and other agar plate cultures), microscopic examination of unstained (e.g. direct faecal smear, Kato-Katz thick smear and formalin ether-concentration method) and stained microscope slides (acid-fast stains, e.g. Kinyoun technique) for parasite identification, and various pathogen-specific tests such as PCR for viruses and diarrhoeagenic E. coli pathotypes, toxin detection kits for $C$. difficile diagnosis, and stool concentration methods for S. stercoralis (e.g. Baermann funnel and Koga agar plate). Examination of more than one stool specimen over consecutive days is crucial, because many intestinal pathogens are irregularly shed in the faeces [149]. 'Classical' approaches to persistent diarrhoea often lead to disappointing results with up to $80 \%$ of cases in which no causative pathogen can be determined [6].

However, even exhaustive laboratory work-up is prone to a host of limitations and challenges that must be considered and addressed. Firstly, gastrointestinal complaints are often caused by non-infectious causes, and a combination of different clinical signs and symptoms as well as further tests are needed to detect and exclude such non-infectious aetiologies. Secondly, available epidemiological data regarding the sought infectious pathogens in the tropics are scarce, thus requiring broad diagnostic testing to avoid overlooking of important pathogens. Thirdly, studies should be carried out in different social-ecological settings to assess the influence of cultural, demographic, genetic, geographic, socioeconomic and health system related factors on predominating pathogens. Fourthly, such research must address all pathogen classes and should not be limited to one-dimensional approaches examining either bacteria or parasites only. Fifthly, there are certain issues unique to gastrointestinal diseases which clearly distinguish them from other organ disorders; most importantly, the finding of a given pathogen may not necessarily mean that the patient's complaints are caused by this organism [179]. Bacteria, helminths and intestinal protozoa may often be found as harmless commensals or even beneficial parts of the gastrointestinal flora, and thus such findings may represent coincidence rather than causality [180-182]. This is of particular importance when different potential pathogens are found concurrently in one faecal specimen and the causative one(s) have to be differentiated. Sixthly, even primarily non-intestinal infectious pathogens may cause gastrointestinal symptoms, as has been reported for HIV infection and even malaria in the tropics, where acute or long-lasting diarrhoea may be the only symptom in up to $20 \%$ of all observed cases $[183,184]$. In contrast, patients may as well start to complain about reduced wellbeing and develop clinical symptoms only some weeks to months after clearance of an intestinal pathogen, as is the case in postinfectious irritable bowel syndrome [185]. Finally, the variety of possible pathogens affecting the gut is so exhaustive that even very sophisticated diagnostic approaches will not be able to detect every pathogen with satisfactory sensitivity and specificity, especially when considering the cost and practical applicability of some specialised techniques that are not currently feasible in most parts of the tropics.

\section{Conclusion}

There is a pressing need for research targeting persistent digestive disorders as a coherent clinical problem rather than as a disconnected collection of pathologies. This would allow the elaboration of evidence-based diagnosistreatment algorithms centred on patients in resourceconstrained settings, where data availability is scarce and patient management often driven by experience and local beliefs. This is the overarching goal of the NIDIAG consortium, focusing on digestive disorders as discussed here, as well as on neurological disorders [23] and persistent fever [24]. Additionally, such investigations will optimise the use of existing diagnostic tests and advance the development of new methods, which are ideally able to concurrently detect a broad spectrum of intestinal pathogens with a high sensitivity and specificity, and which are 
simple and affordable enough to be performed in lowincome countries where prevalences of persistent digestive disorders are generally high. Moreover, the thorough evaluation of reference tests for intestinal pathogens can serve as diagnostic 'gold' standard in the standardisation and validation of easily applicable RDTs, which are highly needed tools in resource-constrained field settings. Finally, such in-depth investigations are not only important for individual patient management, but also for public health policy making (e.g. to assess the efficacy and costeffectiveness of ongoing preventive chemotherapy control programmes targeting helminthiases). There is a need for improved diagnostics for persistent digestive disorders in the tropics. It is desirable to conduct a multicentric study to investigate the clinical presentations and respective identified pathogens of large patient cohorts presenting with non-acute gastrointestinal diseases as a first step towards more reliable and evidence-based clinical case management in the tropics.

\section{Competing interest}

The authors declare that they have no competing interests.

\section{Authors' contributions}

$S L B, J V$ and $J U$ took primary responsibility for the literature search. SLB, JV, SK, MP and JU drafted the manuscript. According to their areas of expertise, the authors critically revised the text chapters (bacteria: SLB, DCW, LVM, CPY and MAM; parasites: SLB, SK, DCW, KP, HM, MS, FM, MAM, LVL, EKN and JU; viruses: MP; clinical aspects: SLB, CPY, JJ, EB and SR). All authors contributed to the manuscript, read and approved the final version.

\section{Financial support}

This work is part of the NIDIAG network (Collaborative Project; http://www. nidiag.org) supported by the European Commission under the Health Cooperation Work Programme of the 7th Framework Programme (grant agreement no. 260260).

\section{Acknowledgements}

We thank Yvette Endriss from the Swiss Tropical and Public Health Institute in Basel, Switzerland, for her help in taking photographs of various helminth eggs for Figure 1.

\section{Author details}

'Department of Epidemiology and Public Health, Swiss Tropical and Public Health Institute, Basel, Switzerland. 'University of Basel, Basel, Switzerland. ${ }^{3}$ Institute of Medical Microbiology and Hygiene, University of Saarland Hospital, Homburg/Saar, Germany. ${ }^{4}$ Department of Virology, University of Freiburg, Freiburg, Germany. ${ }^{5}$ Faculty of Infectious and Tropical Diseases, London School of Hygiene and Tropical Medicine, London, United Kingdom. ${ }^{6}$ Department of Biomedical Sciences, Institute of Tropical Medicine, Antwerp, Belgium. ${ }^{7}$ Department of Medical Services and Diagnostic, Swiss Tropical and Public Health Institute, Basel, Switzerland. ${ }^{8}$ J.D. MacLean Centre for Tropical Diseases, and Divisions of Infectious Diseases and Medical Microbiology, McGill University Health Centre, Montreal, Canada. ${ }^{9}$ Department of Clinical Sciences, Institute of Tropical Medicine, Antwerp, Belgium. ${ }^{10}$ Institut National de Recherche en Santé Publique, Bamako, Mali. ${ }^{11}$ Department of Internal Medicine, B P Koirala Institute of Health Sciences, Dharan, Nepal. ${ }^{12}$ Centre for Tropical Medicine, Faculty of Medicine, Gadjah Mada University, Yogyakarta, Indonesia. ${ }^{13}$ Department of Public Health, Institute of Tropical Medicine, Antwerp, Belgium. ${ }^{14}$ Institut National de Recherche Biomédicale, Kinshasa, Democratic Republic of the Congo. ${ }^{15}$ Universite de Kinshasa, Kinshasa, Democratic Republic of the Congo. ${ }^{16}$ Department of Parasitology, Leiden University Medical Center, Leiden, The Netherlands. ${ }^{17}$ Unité de Formation et de Recherche Biosciences, Université Félix Houphouët-Boigny, Abidjan, Côte d'Ivoire. ${ }^{18}$ Département Environnement et Santé, Centre Suisse de
Recherches Scientifiques en Côte d'Ivoire, Abidjan, Côte d'Ivoire. ${ }^{19}$ Division of Humanitarian and International Medicine, Geneva University Hospitals, Geneva, Switzerland.

Received: 17 September 2012 Accepted: 14 January 2013

Published: 24 January 2013

\section{References}

1. Bartram J, Cairncross S: Hygiene, sanitation, and water: forgotten foundations of health. PLOS Med 2010, 7:e1000367.

2. WHO: Diarrhoeal disease. Fact sheet no. 330. Auqust 2009. Geneva: World Health Organization; 2009.

3. Murray CJL, Vos T, Lozano R, Naghavi M, Flaxman AD, Michaud C, Ezzati M, Shibuya K, Salomon JA, Abdalla S, et al: Disability-adjusted life years (DALYs) for 291 diseases and injuries in 21 regions, 1990-2010: a systematic analysis for the Global Burden of Disease Study 2010. Lancet 2012, 380:2197-2223.

4. Lozano R, Naghavi M, Foreman K, Lim S, Shibuya K, Aboyans V, Abraham J, Adair T, Aggarwal R, Ahn SY, et al: Global and regional mortality from 235 causes of death for 20 age groups in 1990 and 2010: a systematic analysis for the Global Burden of Disease Study 2010. Lancet 2012, 380:2095-2128.

5. Tate JE, Burton AH, Boschi-Pinto C, Steele AD, Duque J, Parashar UD: 2008 estimate of worldwide rotavirus-associated mortality in children younger than 5 years before the introduction of universal rotavirus vaccination programmes: a systematic review and meta-analysis. Lancet Infect Dis 2012, 12:136-141.

6. Pawlowski SW, Warren CA, Guerrant R: Diagnosis and treatment of acute or persistent diarrhea. Gastroenterology 2009, 136:1874-1886.

7. Utzinger J, Becker SL, Knopp S, Blum J, Neumayr AL, Keiser J, Hatz CF: Neglected tropical diseases: diagnosis, clinical management, treatment and control. Swiss Med Wkly 2012, 142:w13727.

8. Pullan RL, Brooker SJ: The global limits and population at risk of soiltransmitted helminth infections in 2010. Parasit Vectors 2012, 5:81.

9. Hotez PJ, Molyneux DH, Fenwick A, Ottesen E, Ehrlich Sachs S, Sachs JD: Incorporating a rapid-impact package for neglected tropical diseases with programs for HIV/AIDS, tuberculosis, and malaria. PLOS Med 2006 3:e102.

10. Utzinger J: A research and development agenda for the control and elimination of human helminthiases. PLoS Negl Trop Dis 2012, 6:e1646.

11. Hotez PJ, Molyneux DH, Fenwick A, Kumaresan J, Ehrlich Sachs S, Sachs JD, Savioli L: Control of neglected tropical diseases. N Engl J Med 2007, 357:1018-1027

12. Hotez P: Hookworm and poverty. Ann N Y Acad Sci 2008, 1136:38-44

13. Hotez PJ, Fenwick A, Savioli L, Molyneux DH: Rescuing the bottom billion through control of neglected tropical diseases. Lancet 2009, 373:1570-1575.

14. Conteh L, Engels T, Molyneux DH: Socioeconomic aspects of neglected tropical diseases. Lancet 2010, 375:239-247.

15. King $\mathrm{CH}$ : Parasites and poverty: the case of schistosomiasis. Acta Trop 2010, 113:95-104.

16. Cavalli A, Bamba SI, Traore MN, Boelaert M, Coulibaly Y, Polman K, Pirard M, Van Dormael M: Interactions between Global Health Initiatives and country health systems: the case of a neglected tropical diseases control program in Mali. PLoS Negl Trop Dis 2010, 4:e798.

17. Marchal B, Van Dormael M, Pirard M, Cavalli A, Kegels G, Polman K: Neglected tropical disease (NTD) control in health systems: the interface between programmes and general health services. Acta Trop 2011, 120(Suppl 1):S177-S185.

18. Amazigo UV, Leak SG, Zoure HG, Njepuome N, Lusamba-Dikassa PS: Community-driven interventions can revolutionise control of neglected tropical diseases. Trends Parasitol 2012, 28:231-238.

19. Robays J, Lefevre P, Lutumba P, Lubanza S, Kande Betu Ku Mesu V, Van der Stuyft P, Boelaert M: Drug toxicity and cost as barriers to community participation in HAT control in the Democratic Republic of Congo. Trop Med Int Health 2007, 12:290-298.

20. Kibadi K, Boelaert M, Kayinua M, Minuku JB, Muyembe-Tamfum JJ, Portaels F, Lefevre $P$ : Therapeutic itineraries of patients with ulcerated forms of Mycobacterium ulcerans (Buruli ulcer) disease in a rural health zone in the Democratic Republic of Congo. Trop Med Int Health 2009, 14:1110-1116. 
21. Brun R, Blum J, Chappuis F, Burri C: Human African trypanosomiasis. Lancet 2010, 375:148-159.

22. Birx $D$, de Souza $M$, Nkengasong JN: Laboratory challenges in the scaling up of HIV, TB, and malaria programs: the interaction of health and laboratory systems, clinical research, and service delivery. Am J Clin Pathol 2009, 131:849-851.

23. Yansouni $C P$, Bottieau $E$, Lutumba $P$, Winkler $A S$, Lynen $L$, Büscher $P$, Jacobs J, Gillet $P$, Lejon V, Alirol E, et al: Rapid diagnostic tests for neurological infections in Central Africa. Lancet Infect Dis 2013, in press.

24. Yansouni $\mathrm{CP}$, Bottieau E, Chappuis F, Phoba MF, Lunguya O, Ifeka BB, Jacobs $\mathrm{J}$ : Rapid diagnostic tests for a coordinated approach to fever syndromes in low-resource settings. Clin Infect Dis 2012, 55:610-611.

25. Lacour M, Zunder T, Huber R, Sander A, Daschner F, Frank U: The pathogenetic significance of intestinal Candida colonization - a systematic review from an interdisciplinary and environmental medical point of view. Int J Hyg Environ Health 2002, 205:257-268.

26. Abba K, Sinfield R, Hart CA, Garner P: Pathogens associated with persistent diarrhoea in children in low and middle income countries: systematic review. BMC Infect Dis 2009, 9:88.

27. Moore SR: Update on prolonged and persistent diarrhea in children Curr Opin Gastroenterol 2011, 27:19-23.

28. Pfeiffer ML, Dupont HL, Ochoa TJ: The patient presenting with acute dysentery - a systematic review. J Infect 2012, 64:374-386.

29. Barratt JL, Harkness J, Marriott D, Ellis JT, Stark D: A review of Dientamoeba fragilis carriage in humans: several reasons why this organism should be considered in the diagnosis of gastrointestinal illness. Gut Microbes 2011, 2:3-12.

30. Scanlan PD: Blastocystis: past pitfalls and future perspectives. Trends Parasitol 2012, 28:327-334.

31. Janda JM, Abbott SL: The genus Aeromonas: taxonomy, pathogenicity, and infection. Clin Microbiol Rev 2010, 23:35-73.

32. Granato PA, Chen L, Holiday I, Rawling RA, Novak-Weekley SM, Quinlan T, Musser KA: Comparison of premier CAMPY enzyme immunoassay (EIA), ProSpecT Campylobacter EIA, and ImmunoCard STAT! CAMPY tests with culture for laboratory diagnosis of Campylobacter enteric infections. J Clin Microbiol 2010, 48:4022-4027.

33. Bartlett JG, Gerding DN: Clinical recognition and diagnosis of Clostridium difficile infection. Clin Infect Dis 2008, 46(Suppl 1):S12-S18.

34. Planche T, Aghaizu A, Holliman R, Riley P, Poloniecki J, Breathnach A, Krishna S: Diagnosis of Clostridium difficile infection by toxin detection kits: a systematic review. Lancet Infect Dis 2008, 8:777-784.

35. Lemee L, Dhalluin A, Testelin S, Mattrat MA, Maillard K, Lemeland JF, Pons $J$ L: Multiplex PCR targeting tpi (triose phosphate isomerase), $t c d A$ (Toxin A), and $t c d B$ (Toxin B) genes for toxigenic culture of Clostridium difficile. J Clin Microbiol 2004, 42:5710-5714.

36. Kaur P, Chakraborti A, Asea A: Enteroaggregative Escherichia coli: an emerging enteric food borne pathogen. Interdiscip Perspect Infect Dis 2010, 2010:254159.

37. Ochoa TJ, Barletta F, Contreras C, Mercado E: New insights into the epidemiology of enteropathogenic Escherichia coli infection. Trans R Soc Trop Med Hyg 2008, 102:852-856.

38. Szakal DD, Schneider G, Pal T: A colony blot immune assay to identify enteroinvasive Escherichia coli and Shigella in stool samples. Diagn Microbiol Infect Dis 2003, 45:165-171.

39. Gould LH, Bopp C, Strockbine N, Atkinson R, Baselski V, Body B, Carey R, Crandall C, Hurd S, Kaplan R, et al: Recommendations for diagnosis of shiga toxin-producing Escherichia coli infections by clinical laboratories. MMWR Recomm Rep 2009, 58:1-14.

40. Hermos CR, Janineh M, Han LL, McAdam AJ: Shiga toxin-producing Escherichia coli in children: diagnosis and clinical manifestations of 0157: H7 and non-0157:H7 infection. J Clin Microbiol 2011, 49:955-959.

41. Nataro JP, Kaper JB: Diarrheagenic Escherichia coli. Clin Microbiol Rev 1998, 11:142-201.

42. Guion CE, Ochoa TJ, Walker CM, Barletta F, Cleary TG: Detection of diarrheagenic Escherichia coli by use of melting-curve analysis and real-time multiplex PCR. J Clin Microbiol 2008, 46:1752-1757.

43. Wiener RS, Della-Latta P, Schluger NW: Effect of nucleic acid amplification for Mycobacterium tuberculosis on clinical decision making in suspected extrapulmonary tuberculosis. Chest 2005, 128:102-107.

44. Giouleme O, Paschos P, Katsaros M, Papalexi F, Karabatsou S, Masmanidou M, Koliouskas D: Intestinal tuberculosis: a diagnostic challenge - case report and review of the literature. Eur J Gastroenterol Hepatol 2011, 23:1074-1077.

45. Bhan MK, Bahl R, Bhatnagar S: Typhoid and paratyphoid fever. Lancet 2005, 366:749-762.

46. Kariuki S: Typhoid fever in sub-Saharan Africa: challenges of diagnosis and management of infections. J Infect Dev Ctries 2008, 2:443-447.

47. Gordon MA: Invasive nontyphoidal Salmonella disease: epidemiology, pathogenesis and diagnosis. Curr Opin Infect Dis 2011, 24:484-489.

48. Niyogi SK: Shigellosis. J Microbiol 2005, 43:133-143.

49. Marth T, Schneider T: Whipple disease. Curr Opin Gastroenterol 2008, 24:141-148

50. Shears P: Recent developments in cholera. Curr Opin Infect Dis 2001, 14:553-558.

51. Neogi SB, Chowdhury N, Asakura M, Hinenoya A, Haldar S, Saidi SM, Kogure K, Lara RJ, Yamasaki S: A highly sensitive and specific multiplex PCR assay for simultaneous detection of Vibrio cholerae, Vibrio parahaemolyticus and Vibrio vulnificus. Lett Appl Microbiol 2010, 51:293-300.

52. Bockemühl J, Roggentin P: Intestinal yersiniosis. Clinical importance, epidemiology, diagnosis, and prevention. Bundesgesundheitsblatt Gesundheitsforschung Gesundheitsschutz 2004, 47:685-691. (in German).

53. M'lkanatha NM, Dettinger LA, Perry A, Rogers P, Reynolds SM, Nachamkin I: Culturing stool specimens for Campylobacter spp., Pennsylvania, USA. Emerg Infect Dis 2012, 18:484-487.

54. Shanholtzer CJ, Peterson LR, Olson MN, Gerding DN: Prospective study of gram-stained stool smears in diagnosis of Clostridium difficile colitis. J Clin Microbiol 1983, 17:906-908.

55. Schuster FL, Ramirez-Avila L: Current world status of Balantidium coli. Clin Microbiol Rev 2008, 21:626-638.

56. Stark D, van Hal S, Marriott D, Ellis J, Harkness J: Irritable bowel syndrome: a review on the role of intestinal protozoa and the importance of their detection and diagnosis. Int J Parasitol 2007, 37:11-20

57. Stensvold CR, Nielsen HV, Molbak K, Smith HV: Pursuing the clinical significance of Blastocystis - diagnostic limitations. Trends Parasitol 2009, 25:23-29.

58. Zhang X, Qiao J, Wu X, Da R, Zhao L, Wei Z: In vitro culture of Blastocystis hominis in three liquid media and its usefulness in the diagnosis of blastocystosis. Int J Infect Dis 2012, 16:e23-e28.

59. Verweij JJ, Blange RA, Templeton $K$, Schinkel J, Brienen EA, van Rooyen MA van Lieshout L, Polderman AM: Simultaneous detection of Entamoeba histolytica, Giardia lamblia, and Cryptosporidium parvum in fecal samples by using multiplex real-time PCR. J Clin Microbiol 2004, 42:1220-1223.

60. van Lieshout L, Verweij JJ: Newer diagnostic approaches to intestinal protozoa. Curr Opin Infect Dis 2010, 23:488-493.

61. Caccio SM, Pozio E: Advances in the epidemiology, diagnosis and treatment of cryptosporidiosis. Expert Rev Anti Infect Ther 2006, 4:429-443.

62. Davies AP, Chalmers RM: Cryptosporidiosis. BMJ 2009, 339:b4168

63. Ortega YR, Sanchez R: Update on Cyclospora cayetanensis, a food-borne and waterborne parasite. Clin Microbiol Rev 2010, 23:218-234.

64. Verweij JJ, Mulder B, Poell B, van Middelkoop D, Brienen EA, van Lieshout L: Real-time PCR for the detection of Dientamoeba fragilis in fecal samples. Mol Cell Probes 2007, 21:400-404.

65. Stark D, Barratt J, Roberts T, Marriott D, Harkness J, Ellis J: Comparison of microscopy, two xenic culture techniques, conventional and real-time PCR for the detection of Dientamoeba fragilis in clinical stool samples. Eur J Clin Microbiol Infect Dis 2010, 29:411-416.

66. Qvarnstrom $Y$, James C, Xayavong M, Holloway BP, Visvesvara GS, Sriram R, da Silva AJ: Comparison of real-time PCR protocols for differential laboratory diagnosis of amebiasis. J Clin Microbiol 2005, 43:5491-5497.

67. Fotedar R, Stark D, Beebe N, Marriott D, Ellis J, Harkness J: Laboratory diagnostic techniques for Entamoeba species. Clin Microbiol Rev 2007, 20:511-532.

68. Clark CG, Diamond LS: Methods for cultivation of luminal parasitic protists of clinical importance. Clin Microbiol Rev 2002, 15:329-341.

69. Kebede A, Verweij J, Dorigo-Zetsma W, Sanders E, Messele T, van Lieshout L, Petros B, Polderman T: Overdiagnosis of amoebiasis in the absence of Entamoeba histolytica among patients presenting with diarrhoea in Wonji and Akaki, Ethiopia. Trans R Soc Trop Med Hyg 2003, 97:305-307.

70. Kebede A, Verweij JJ, Petros B, Polderman AM: Misleading microscopy in amoebiasis. Trop Med Int Health 2004, 9:651-652. 
71. Nagaty IM, Hegazi MM: Dot-ELISA copro-antigen and direct stool examination in diagnosis of giardiasis patients. J Egypt Soc Parasitol 2007 37:641-648.

72. ten Hove RJ, van Lieshout L, Brienen EA, Perez MA, Verweij JJ: Real-time polymerase chain reaction for detection of Isospora belli in stool samples. Diagn Microbiol Infect Dis 2008, 61:280-283.

73. Murphy SC, Hoogestraat DR, Sengupta DJ, Prentice J, Chakrapani A, Cookson BT: Molecular diagnosis of cystoisosporiasis using extendedrange PCR screening. J Mol Diagn 2011, 13:359-362.

74. Didier ES, Weiss LM: Microsporidiosis: not just in AIDS patients. Curr Opin Infect Dis 2011, 24:490-495.

75. Didier ES, Weiss LM: Microsporidiosis: current status. Curr Opin Infect Dis 2006, 19:485-492.

76. Verweij JJ, Ten Hove R, Brienen EA, van Lieshout L: Multiplex detection of Enterocytozoon bieneusi and Encephalitozoon spp. in fecal samples using real-time PCR. Diagn Microbiol Infect Dis 2007, 57:163-167.

77. Scholz T, Garcia HH, Kuchta R, Wicht B: Update on the human broad tapeworm (genus diphyllobothrium), including clinical relevance. Clin Microbiol Rev 2009, 22:146-160.

78. Wicht B, Yanagida T, Scholz T, Ito A, Jimenez JA, Brabec J: Multiplex PCR for differential identification of broad tapeworms (Cestoda: Diphyllobothrium) infecting humans. J Clin Microbiol 2010, 48:3111-3116.

79. Steinmann P, Cringoli G, Bruschi F, Matthys B, Lohourignon LK, Castagna B, Maurelli MP, Morgoglione ME, Utzinger J, Rinaldi L: FLOTAC for the diagnosis of Hymenolepis spp. infection: proof-of-concept and comparing diagnostic accuracy with other methods. Parasitol Res 2012, 111:749-754.

80. Brunetti $E$, White AC Jr: Cestode infestations: hydatid disease and cysticercosis. Infect Dis Clin North Am 2012, 26:421-435.

81. Katz N, Chaves A, Pellegrino J: A simple device for quantitative stool thicksmear technique in schistosomiasis mansoni. Rev Inst Med Trop São Paulo 1972 14:397-400.

82. WHO: Bench aids for the diagnosis of intestinal parasites. Geneva: World Health Organization; 1994.

83. Basuni M, Muhi J, Othman N, Verweij JJ, Ahmad M, Miswan N, Rahumatullah A, Aziz FA, Zainudin NS, Noordin R: A pentaplex real-time polymerase chain reaction assay for detection of four species of soil-transmitted helminths. AmJTrop Med Hyg 2011, 84:338-343.

84. Soukhathammavong P, Sayasone S, Harimanana AN, Akkhavong A, Thammasack S, Phoumindr N, Choumlivong K, Keoluangkhot V, Phongmany S, Akkhavong K, et al: Three cases of intestinal capillariasis in Lao People's Democratic Republic. AmJTrop Med Hyg 2008, 79:735-738.

85. Attia RA, Tolba ME, Yones DA, Bakir HY, Eldeek HE, Kamel S: Capillaria philippinensis in Upper Egypt: has it become endemic? AmJTrop Med Hyg 2012, 86:126-133.

86. Montes M, Sawhney C, Barros N: Strongyloides stercoralis: there but not seen. Curr Opin Infect Dis 2010, 23:500-504.

87. Verweij JJ, Canales M, Polman K, Ziem J, Brienen EA, Polderman AM, van Lieshout L: Molecular diagnosis of Strongyloides stercoralis in faecal samples using real-time PCR. Trans R Soc Trop Med Hyg 2009, 103:342-346.

88. Keiser J, Duthaler U, Utzinger J: Update on the diagnosis and treatment of food-borne trematode infections. Curr Opin Infect Dis 2010, 23:513-520.

89. Glinz D, Silué KD, Knopp S, Lohourignon LK, Yao KP, Steinmann P, Rinaldi L, Cringoli G, N'Goran EK, Utzinger J: Comparing diagnostic accuracy of Kato-Katz, Koga agar plate, ether-concentration, and FLOTAC for Schistosoma mansoni and soil-transmitted helminths. PLoS Negl Trop Dis 2010, 4:e754.

90. Henke-Gendo C, Ganzenmueller T, Kluba J, Harste G, Raggub L, Heim A: Improved quantitative PCR protocols for adenovirus and CMV with an internal inhibition control system and automated nucleic acid isolation. J Med Virol 2012, 84:890-896.

91. van Maarseveen NM, Wessels E, de Brouwer CS, Vossen AC, Claas EC: Diagnosis of viral gastroenteritis by simultaneous detection of adenovirus group F, astrovirus, rotavirus group a, norovirus genogroups I and II, and sapovirus in two internally controlled multiplex real-time PCR assays. J Clin Virol 2010, 49:205-210.

92. Kantola K, Sadeghi M, Antikainen J, Kirveskari J, Delwart E, Hedman K, Soderlund-Venermo M: Real-time quantitative PCR detection of four human bocaviruses. J Clin Microbiol 2010, 48:4044-4050.

93. Dare RK, Fry AM, Chittaganpitch M, Sawanpanyalert P, Olsen SJ, Erdman DD: Human coronavirus infections in rural Thailand: a comprehensive study using real-time reverse-transcription polymerase chain reaction assays. $J$ Infect Dis 2007, 196:1321-1328.

94. Dierssen U, Rehren F, Henke-Gendo C, Harste G, Heim A: Rapid routine detection of enterovirus RNA in cerebrospinal fluid by a one-step realtime RT-PCR assay. J Clin Virol 2008, 42:58-64.

95. Center for Disease Control and Prevention: Guidelines for appropriate evaluations of HIV testing technologies in Africa. Available at: http://www. who.int/diagnostics_laboratory/publications/EN_HIVEval_Guide.pdf (accessed online: 26 December 2012).

96. Branson BM: The future of HIV testing. J Acquir Immune Defic Syndr 2010, 55(Suppl 2):S102-S105

97. Baumgarte S, de Souza Luna LK, Grywna K, Panning M, Drexler JF, Karsten C, Huppertz HI, Drosten C: Prevalence, types, and RNA concentrations of human parechoviruses, including a sixth parechovirus type, in stool samples from patients with acute enteritis. J Clin Microbiol 2008, 46:242-248.

98. Martiny D, Busson L, Wybo I, El Haj RA, Dediste A, Vandenberg O: Comparison of the Microflex LT and Vitek MS systems for routine identification of bacteria by matrix-assisted laser desorption ionizationtime of flight mass spectrometry. J Clin Microbiol 2012, 50:1313-1325.

99. Bessede E, Delcamp A, Sifre E, Buissonniere A, Megraud F: New methods for detection of campylobacters in stool samples in comparison to culture. J Clin Microbiol 2011, 49:941-944.

100. Kuhn KG, Falkenhorst G, Ceper T, Dalby T, Ethelberg S, Molbak K, Krogfelt KA: Detection of antibodies to Campylobacter in humans using enzymelinked immunosorbent assays: a review of the literature. Diagn Microbiol Infect Dis 2012, 74:113-118.

101. Yaqing H, Wenping Z, Zhiyi Y, Xionghu W, Shouyi Y, Hong Y, Yingchun D, Guifang $\mathrm{H}$ : Detection of human Enterovirus 71 reverse transcription loop-mediated isothermal amplification (RT-LAMP). Lett Appl Microbiol 2012, 54:233-239.

102. Yamazaki W: Sensitive and rapid detection of Campylobacter jejuni and Campylobacter coli using loop-mediated isothermal amplification. Methods Mol Biol 2013, 943:267-277.

103. Parry CM, Wijedoru L, Arjyal A, Baker S: The utility of diagnostic tests for enteric fever in endemic locations. Expert Rev Anti Infect Ther 2011, 9:711-725.

104. Gilman RH, Terminel M, Levine MM, Hernandez-Mendoza P, Hornick RB: Relative efficacy of blood, urine, rectal swab, bone-marrow, and rose-spot cultures for recovery of Salmonella typhi in typhoid fever. Lancet 1975 , 305:1211-1213.

105. Baker S, Favorov M, Dougan G: Searching for the elusive typhoid diagnostic. BMC Infect Dis 2010, 10:45

106. Joost I, Speck K, Herrmann M, von Müller L: Characterisation of Clostridium difficile isolates by sIpA and $t c d C$ gene sequencing. Int J Antimicrob Agents 2009, 33(Suppl 1):S13-S18.

107. Bartlett JG: Clostridium difficile: progress and challenges. Ann N Y Acad Sci 2010, 1213:62-69.

108. de Jong E, de Jong AS, Bartels CJ, Van Der Rijt-Van Den Biggelaar C, Melchers WJ, Sturm PD: Clinical and laboratory evaluation of a real-time PCR for Clostridium difficile toxin A and B genes. Eur J Clin Microbiol Infect Dis 2012, 31:2219-2225.

109. Reller ME, Lema CA, Perl TM, Cai M, Ross TL, Speck KA, Carroll KC: Yield of stool culture with isolate toxin testing versus a two-step algorithm including stool toxin testing for detection of toxigenic Clostridium difficile. J Clin Microbiol 2007, 45:3601-3605.

110. Crobach MJ, Dekkers OM, Wilcox MH, Kuijper EJ: European Society of Clinical Microbiology and Infectious Diseases (ESCMID): data review and recommendations for diagnosing Clostridium difficile infection (CDI). Clin Microbiol Infect 2009, 15:1053-1066.

111. Robert-Koch-Institut: Abschließende Darstellung und Bewertung der epidemiologischen Erkenntnisse im EHEC 0104:H4 Ausbruch Deutschland 2011. Berlin: Robert-Koch-Institut, 2011. (in German). Available at: http://www.rki. de/DE/Content/InfAZ/E/EHEC/EHEC-Abschlussbericht.html (accessed online: 30 August 2012).

112. Donoghue HD, Holton J: Intestinal tuberculosis. Curr Opin Infect Dis 2009, 22:490-496

113. Jeyanathan M, Boutros-Tadros O, Radhi J, Semret M, Bitton A, Behr MA Visualization of mycobacterium avium in Crohn's tissue by oil-immersion microscopy. Microbes Infect 2007, 9:1567-1573.

114. Yu H, Liu Y, Wang Y, Peng L, Li A, Zhang Y: Clinical, endoscopic and histological differentiations between Crohn's disease and intestinal tuberculosis. Digestion 2012, 85:202-209. 
115. Honscha G, Von Groll A, Valenca M, Ramos DF, Sanchotene K, Scaini CJ, Ribeiro MO, da Silva PE: The laboratory as a tool to qualify tuberculosis diagnosis. Int J Tuberc Lung Dis 2008, 12:218-220.

116. Yardley $\mathrm{JH}$, Hendrix TR: Combined electron and light microscopy in Whipple's disease. Demonstration of "bacillary bodies" in the intestine. Bull Johns Hopkins Hosp 1961, 109:80-98.

117. Raoult D, Birg ML, La Scola B, Fournier PE, Enea M, Lepidi H, Roux V, Piette JC, Vandenesch F, Vital-Durand D, et al: Cultivation of the bacillus of Whipple's disease. N Engl J Med 2000, 342:620-625.

118. Raoult D, Fenollar F, Birg ML: Culture of $T$. whipplei from the stool of a patient with Whipple's disease. N Engl J Med 2006, 355:1503-1505.

119. Dobell C: The discovery of the intestinal protozoa of man. Proc $R$ Soc Med 1920, 13:1-15

120. Allen AVH, Ridley DS: Further observations on the formol-ether concentration technique for faecal parasites. J Clin Pathol 1970, 23:545-546

121. Utzinger J, Botero-Kleiven S, Castelli F, Chiodini PL, Edwards H, Kohler N, Gulletta M, Lebbad M, Manser M, Matthys B, et al: Microscopic diagnosis of sodium acetate-acetic acid-formalin-fixed stool samples for helminths and intestinal protozoa: a comparison among European reference laboratories. Clin Microbiol Infect 2010, 16:267-273.

122. Becker SL, Lohourignon LK, Speich B, Rinaldi L, Knopp S, N'Goran EK, Cringoli G, Utzinger J: Comparison of the Flotac-400 dual technique and the formalin-ether concentration technique for diagnosis of human intestinal protozoon infection. J Clin Microbiol 2011, 49:2183-2190.

123. Sharp SE, Suarez CA, Duran Y, Poppiti RJ: Evaluation of the Triage Micro Parasite Panel for detection of Giardia lamblia, Entamoeba histolytical Entamoeba dispar, and Cryptosporidium parvum in patient stool specimens. J Clin Microbiol 2001, 39:332-334.

124. Chalmers RM, Davies AP: Clinical cryptosporidiosis. Exp Parasitol 2010 124:138-146.

125. Diamond LS, Clark CG: A redescription of Entamoeba histolytica Schaudinn, 1903 (Emended Walker, 1911) separating it from Entamoeba dispar Brumpt, 1925. J Eukaryot Microbiol 1993, 40:340-344.

126. Haque R, Neville LM, Hahn P, Petri WA Jr: Rapid diagnosis of Entamoeba infection by using Entamoeba and Entamoeba histolytica stool antigen detection kits. J Clin Microbiol 1995, 33:2558-2561.

127. Fotedar R, Stark D, Beebe N, Marriott D, Ellis J, Harkness J: PCR detection of Entamoeba histolytica, Entamoeba dispar, and Entamoeba moshkovskii in stool samples from Sydney, Australia. J Clin Microbiol 2007, 45:1035-1037.

128. Ximenez C, Moran P, Rojas L, Valadez A, Gomez A: Reassessment of the epidemiology of amebiasis: state of the art. Infect Genet Evol 2009, 9:1023-1032.

129. ten Hove RJ, van Esbroeck M, Vervoort T, van den Ende J, van Lieshout L, Verweij JJ: Molecular diagnostics of intestinal parasites in returning travellers. Eur J Clin Microbiol Infect Dis 2009, 28:1045-1053.

130. de Vlas SJ, Gryseels B: Underestimation of Schistosoma mansoni prevalences. Parasitol Today 1992, 8:274-277.

131. Booth M, Vounatsou P, N'Goran EK, Tanner M, Utzinger J: The influence of sampling effort and the performance of the Kato-Katz technique in diagnosing Schistosoma mansoni and hookworm co-infections in rural Côte d'Ivoire. Parasitology 2003, 127:525-531.

132. Speich B, Knopp S, Mohammed KA, Khamis IS, Rinaldi L, Cringoli G Rollinson D, Utzinger J: Comparative cost assessment of the Kato-Katz and FLOTAC techniques for soil-transmitted helminth diagnosis in epidemiological surveys. Parasit Vectors 2010, 3:71

133. García LS, Bruckner DA: Diagnostic medical parasitology. Washington D.C: ASM Press; 2001.

134. Tarafder MR, Carabin H, Joseph L, Balolong E Jr, Olveda R, McGarvey ST: Estimating the sensitivity and specificity of Kato-Katz stool examination technique for detection of hookworms, Ascaris lumbricoides and Trichuris trichiura infections in humans in the absence of a 'gold standard'. Int Parasitol 2010, 40:399-404.

135. Knopp S, Mgeni AF, Khamis IS, Steinmann P, Stothard JR, Rollinson D, Mart $H$, Utzinger J: Diagnosis of soil-transmitted helminths in the era of preventive chemotherapy: effect of multiple stool sampling and use of different diagnostic techniques. PLoS Negl Trop Dis 2008, 2:e331.

136. Berhe N, Medhin G, Erko B, Smith T, Gedamu S, Bereded D, Moore R, Habte E, Redda A, Gebre-Michael T, et al: Variations in helminth faecal egg counts in Kato-Katz thick smears and their implications in assessing infection status with Schistosoma mansoni. Acta Trop 2004, 92:205-212.
137. Goodman D, Haji HJ, Bickle QD, Stoltzfus RJ, Tielsch JM, Ramsan M, Savioli L, Albonico M: A comparison of methods for detecting the eggs of Ascaris, Trichuris, and hookworm in infant stool, and the epidemiology of infection in Zanzibari infants. Am J Trop Med Hyg 2007, 76:725-731.

138. Steinmann P, Du ZW, Wang LB, Wang XZ, Jiang JY, Li LH, Marti H, Zhou XN, Utzinger J: Extensive multiparasitism in a village of Yunnan province, People's Republic of China, revealed by a suite of diagnostic methods. Am J Trop Med Hyg 2008, 78:760-769.

139. Knopp S, Rinaldi L, Khamis IS, Stothard JR, Rollinson D, Maurelli MP, Steinmann $P$, Marti $H$, Cringoli $G$, Utzinger J: A single FLOTAC is more sensitive than triplicate Kato-Katz for the diagnosis of low-intensity soiltransmitted helminth infections. Trans R Soc Trop Med Hyg 2009, 103:347-354.

140. Steinmann P, Utzinger J, Du ZW, Zhou XN: Multiparasitism: a neglected reality on global, regional and local scale. Adv Parasitol 2010, 73:21-50.

141. Olsen A, van Lieshout L, Marti H, Polderman T, Polman K, Steinmann P, Stothard R, Thybo S, Verweij JJ, Magnussen P: Strongyloidiasis - the most neglected of the neglected tropical diseases? Trans $R$ Soc Trop Med Hyg 2009, 103:967-972.

142. Verweij JJ, van Lieshout L: Intestinal parasitic infections in an industrialized country; a new focus on children with better DNA-based diagnostics. Parasitology 2011, 138:1492-1498.

143. Becker SL, Sieto B, Silué KD, Adjossan L, Kone S, Hatz C, Kern WW, N'Goran EK, Utzinger J: Diagnosis, clinical features, and self-reported morbidity of Strongyloides stercoralis and hookworm infection in a co-endemic setting. PLoS Negl Trop Dis 2011, 5:e1292.

144. World Gastroenterology Organization: WGO practice guideline. Management of strongyloidiasis. Available at: http://www.worldgastroenterology.org/ assets/downloads/en/pdf/guidelines/15_management_strongyloidiasis_en pdf (accessed online: 1 August 2012).

145. Koga K, Kasuya S, Khamboonruang C, Sukhavat K, leda M, Takatsuka N, Kita $\mathrm{K}$, Ohtomo H: A modified agar plate method for detection of Strongyloides stercoralis. Am J Trop Med Hyg 1991, 45:518-521.

146. Feely NM, Waghorn DJ, Dexter T, Gallen I, Chiodini P: Strongyloides stercoralis hyperinfection: difficulties in diagnosis and treatment. Anaesthesia 2010, 65:298-301.

147. Kramme S, Nissen N, Soblik H, Erttmann K, Tannich E, Fleischer B, Panning $M$, Brattig N: Novel real-time PCR for the universal detection of Strongyloides species. J Med Microbiol 2011, 60:454-458.

148. Engels D, Sinzinkayo E, Gryseels B: Day-to-day egg count fluctuation in Schistosoma mansoni infection and its operational implications. Am J Trop Med Hyg 1996, 54:319-324.

149. Utzinger J, Booth M, N'Goran EK, Müller I, Tanner M, Lengeler C: Relative contribution of day-to-day and intra-specimen variation in faecal egg counts of Schistosoma mansoni before and after treatment with praziquantel. Parasitology 2001, 122:537-544.

150. Midzi N, Butterworth AE, Mduluza T, Munyati S, Deelder AM, van Dam GJ: Use of circulating cathodic antigen strips for the diagnosis of urinary schistosomiasis. Trans R Soc Trop Med Hyg 2009, 103:45-51.

151. Clerinx J, Van Gompel A: Schistosomiasis in travellers and migrants. Travel Med Infect Dis 2011, 9:6-24.

152. Coulibaly JT, Knopp S, N'Guessan NA, Silué KD, Fürst T, Lohourignon LK, Brou JK, N'Gbesso YK, Vounatsou P, N'Goran EK, et al: Accuracy of urine circulating cathodic antigen (CCA) test for Schistosoma mansoni diagnosis in different settings of Côte d'Ivoire. PLoS Negl Trop Dis 2011 5:e1384.

153. Shane HL, Verani JR, Abudho B, Montgomery SP, Blackstock AJ, Mwinzi PN, Butler SE, Karanja DM, Secor WE: Evaluation of urine CCA assays for detection of Schistosoma mansoni infection in Western Kenya. PLoS Negl Trop Dis 2011, 5:e951

154. ten Hove RJ, Verweij JJ, Vereecken K, Polman K, Dieye L, van Lieshout L: Multiplex real-time PCR for the detection and quantification of Schistosoma mansoni and S. haematobium infection in stool samples collected in northern Senegal. Trans R Soc Trop Med Hyg 2008, 102:179-185.

155. Obeng BB, Aryeetey YA, de Dood CJ, Amoah AS, Larbi IA, Deelder AM, Yazdanbakhsh M, Hartgers FC, Boakye DA, Verweij JJ, et al: Application of a circulating-cathodic-antigen (CCA) strip test and real-time PCR, in comparison with microscopy, for the detection of Schistosoma haematobium in urine samples from Ghana. Ann Trop Med Parasitol 2008, 102:625-633.

156. Wardlaw T, Salama P, Brocklehurst C, Chopra M, Mason E: Diarrhoea: why children are still dying and what can be done. Lancet 2010, 375:870-872. 
157. Clark B, McKendrick M: A review of viral gastroenteritis. Curr Opin Infect Dis 2004, 17:461-469.

158. Soares CC, Volotao EM, Albuquerque MC, da Silva FM, de Carvalho TR, Nozawa CM, Linhares RE, Santos N: Prevalence of enteric adenoviruses among children with diarrhea in four Brazilian cities. J Clin Virol 2002, 23:171-177.

159. Seto D, Chodosh J, Brister JR, Jones MS: Using the whole-genome sequence to characterize and name human adenoviruses. J Virol 2011, 85:5701-5702

160. Grandien M, Pettersson CA, Svensson L, Uhnoo I: Latex agglutination test for adenovirus diagnosis in diarrheal disease. J Med Virol 1987, 23:311-316.

161. Gleaves CA, Militoni J, Ashley RL: An enzyme immunoassay for the direct detection of adenovirus in clinical specimens. Diagn Microbiol Infect Dis 1993, 17:57-59.

162. Caballero S, Guix S, El-Senousy WM, Calico I, Pinto RM, Bosch A: Persistent gastroenteritis in children infected with astrovirus: association with serotype-3 strains. J Med Virol 2003, 71:245-250

163. Dalton RM, Roman ER, Negredo AA, Wilhelmi ID, Glass RI, Sanchez-Fauquier A: Astrovirus acute gastroenteritis among children in Madrid, Spain. Pediatr Infect Dis J 2002, 21:1038-1041

164. Jartti T, Hedman K, Jartti L, Ruuskanen O, Allander T, Soderlund-Venermo M: Human bocavirus - the first 5 years. Rev Med Virol 2012, 22:46-64.

165. Don M, Soderlund-Venermo M, Hedman K, Ruuskanen O, Allander T, Korppi M: Don't forget serum in the diagnosis of human bocavirus infection. J Infect Dis 2011, 203:1031-1032. author reply 1032-1033.

166. Green KY: Caliciviridae: the noroviruses. In Fields virology. 5th edition. Edited by Knipe D, et al. Philadelphia, PA, USA: Lippincott Williams \& Wilkins; 2007:949-979.

167. Burton-MacLeod JA, Kane EM, Beard RS, Hadley LA, Glass RI, Ando T: Evaluation and comparison of two commercial enzyme-linked immunosorbent assay kits for detection of antigenically diverse human noroviruses in stool samples. J Clin Microbiol 2004, 42:2587-2595.

168. Morillo SG, Luchs A, Cilli A, Ribeiro CD, Calux SJ, Carmona Rde C, Timenetsky Mdo C: Norovirus 3rd Generation kit: an improvement for rapid diagnosis of sporadic gastroenteritis cases and valuable for outbreak detection. J Virol Meth 2011, 173:13-16.

169. Gunson RN, Collins TC, Carman WF: The real-time detection of sapovirus. J Clin Virol 2006, 35:321-322.

170. Esper F, Ou Z, Huang YT: Human coronaviruses are uncommon in patients with gastrointestinal illness. J Clin Virol 2010, 48:131-133.

171. Baroco AL, Oldfield EC: Gastrointestinal cytomegalovirus disease in the immunocompromised patient. Curr Gastroenterol Rep 2008, 10:409-416.

172. Nasri D, Bouslama L, Pillet S, Bourlet T, Aouni M, Pozzetto B: Basic rationale, current methods and future directions for molecular typing of human enterovirus. Expert Rev Mol Diagn 2007, 7:419-434

173. Tapia G, Cinek O, Witso E, Kulich M, Rasmussen T, Grinde B, Ronningen KS: Longitudinal observation of parechovirus in stool samples from Norwegian infants. J Med Virol 2008, 80:1835-1842.

174. Harvala H, Simmonds P: Human parechoviruses: biology, epidemiology and clinical significance. J Clin Virol 2009, 45:1-9.

175. Wolffs PF, Bruggeman CA, van Well GT, van Loo IH: Replacing traditional diagnostics of fecal viral pathogens by a comprehensive panel of real-time PCRs. J Clin Microbiol 2011, 49:1926-1931.

176. Pang XL, Lee B, Boroumand N, Leblanc B, Preiksaitis JK, Yu Ip CC: Increased detection of rotavirus using a real time reverse transcription-polymerase chain reaction (RT-PCR) assay in stool specimens from children with diarrhea. J Med Virol 2004, 72:496-501.

177. Jothikumar N, Kang G, Hill VR: Broadly reactive TaqMan assay for real-time RT-PCR detection of rotavirus in clinical and environmental samples. J Virol Meth 2009, 155:126-131.

178. Bhaijee F, Subramony C, Tang SJ, Pepper DJ: Human immunodeficiency virus-associated gastrointestinal disease: common endoscopic biopsy diagnoses. Patholog Res Int 2011, 2011:247923.

179. Lowe AM, Yansouni CP, Behr MA: Causality and gastrointestinal infections: Koch, Hill, and Crohn's. Lancet Infect Dis 2008, 8:720-726.

180. Zimmermann MB, Chassard C, Rohner F, N'Goran EK, Nindjin C, Dostal A, Utzinger J, Ghattas H, Lacroix C, Hurrell RF: The effects of iron fortification on the gut microbiota in African children: a randomized controlled trial in Côte d'Ivoire. Am J Clin Nutr 2010, 92:1406-1415.
181. Nicholson JK, Holmes E, Kinross J, Burcelin R, Gibson G, Jia W, Pettersson S: Host-gut microbiota metabolic interactions. Science 2012, 336:1262-1267.

182. Clemente JC, Ursell LK, Parfrey LW, Knight R: The impact of the gut microbiota on human health: an integrative view. Cell 2012, 148:1258-1270.

183. Prasad RN, Virk KJ: Malaria as a cause of diarrhoea - a review. P N G Med J 1993, 36:337-341.

184. Reisinger EC, Fritzsche C, Krause R, Krejs GJ: Diarrhea caused by primarily non-gastrointestinal infections. Nat Clin Pract Gastroenterol Hepatol 2005, 2:216-222.

185. Spiller R, Garsed K: Postinfectious irritable bowel syndrome. Gastroenterology 2009, 136:1979-1988.

doi:10.1186/1471-2334-13-37

Cite this article as: Becker et al:: Persistent digestive disorders in the tropics: causative infectious pathogens and reference diagnostic tests. BMC Infectious Diseases 2013 13:37.

\section{Submit your next manuscript to BioMed Central and take full advantage of:}

- Convenient online submission

- Thorough peer review

- No space constraints or color figure charges

- Immediate publication on acceptance

- Inclusion in PubMed, CAS, Scopus and Google Scholar

- Research which is freely available for redistribution 